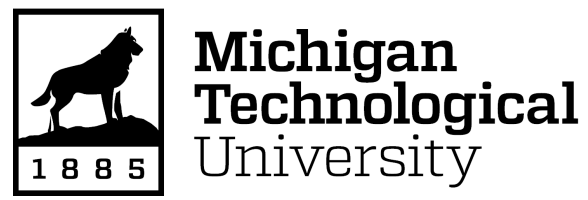

Michigan Technological University Digital Commons @ Michigan Tech

3-31-2015

\title{
Disdrometer network observations of finescale spatial-temporal clustering in rain
}

\author{
A. R. Jameson \\ RJH Scientific, Inc., El Cajon, California \\ M. L. Larsen \\ College of Charleston \\ A. Kostinski \\ Michigan Technological University
}

Follow this and additional works at: https://digitalcommons.mtu.edu/physics-fp

Part of the Physics Commons

\section{Recommended Citation}

Jameson, A. R., Larsen, M. L., \& Kostinski, A. (2015). Disdrometer network observations of finescale spatial-temporal clustering in rain. Journal of the Atmospheric Sciences, 76(3), 1648-1666.

http://dx.doi.org/10.1175/JAS-D-14-0136.1

Retrieved from: https://digitalcommons.mtu.edu/physics-fp/176

Follow this and additional works at: https://digitalcommons.mtu.edu/physics-fp

Part of the Physics Commons 


\title{
Disdrometer Network Observations of Finescale Spatial-Temporal Clustering in Rain
}

\author{
A. R. JAMESON \\ RJH Scientific, Inc., El Cajon, California \\ M. L. LARSEN \\ College of Charleston, Charleston, South Carolina \\ A. B. KOSTINSKI \\ Michigan Technological University, Houghton, Michigan \\ (Manuscript received 12 May 2014, in final form 6 November 2014)

\begin{abstract}
The spatial clustering of drops is a defining characteristic of rain on all scales from centimeters to kilometers. It is the physical basis for much of the observed variability in rain. The authors report here on the temporal-spatial 1-min counts using a network of 21 optical disdrometers over a small area near Charleston, South Carolina. These observations reveal significant differences between spatial and temporal structures (i.e., clustering) for different sizes of drops, which suggest that temporal observations of clustering cannot be used to infer spatial clustering simply using by an advection velocity as has been done in past studies. It is also shown that both spatial and temporal clustering play a role in rain variability depending upon the drop size. The more convective rain is dominated by spatial clustering while the opposite holds for the more stratiform rain.

Like previous time series measurements by a single disdrometer but in contradiction with widely accepted drop size distribution power-law relations, it is also shown that there is a linear relation between 1-min averages of the rainfall rate $R$ over the network and the average total number of drops $N_{t}$. However, the network (area) $R-N_{t}$ relation differs from those derived strictly from time series observations by individual disdrometers. These differences imply that the temporal and spatial size distributions and their variabilities are not equivalent.
\end{abstract}

\section{Introduction}

That rain is spatially variable is well recognized (e.g., Krajewski et al. 2003; Koutsoyiannis 2006; Molini et al. 2009; Smith et al. 2009; Jaffrain et al. 2011; Jaffrain and Berne 2012). While there have been important strides in the development of a broad spectral statistical framework for treating the spatial-temporal statistical characterization of some bulk parameters such as the rainfall rate (Kundu and Travis 2013), no such framework is possible at the level of individual raindrops without vastly improved observations. The humble purpose of this study is simply to present some observations that we hope will contribute toward reaching that goal one day.

Corresponding author address: A. R. Jameson, 5625 N. 32nd St., Arlington, VA 22207-1560.

E-mail: arjatrjhsci@verizon.net
In particular, none of the studies just mentioned approach the physical origins of this variability as a problem in statistical physics on the level of individual drops. Little is known about the spatial distribution of raindrops of various sizes themselves, particularly over scales of less than $100 \mathrm{~m}$. Yet the existence of small-scale drop structures on meter scales is not surprising to anyone who has watched rain from a thunderstorm (Fig. 1a), particularly as it sweeps across the pavement (Fig. 1b). The white streak extending several meters in length and 1 or $2 \mathrm{~m}$ wide across the darker road is one example of a patch of larger drops that splashes, producing an optically reflective cloud of drop fragments. Aside from suggesting a very dynamic view of the meaning of a drop size distribution (DSD) rather than the stagnant one usually considered (Jameson and Kostinski 2000), this particular patch was observed by the lead author to sweep across the road in a matter of 

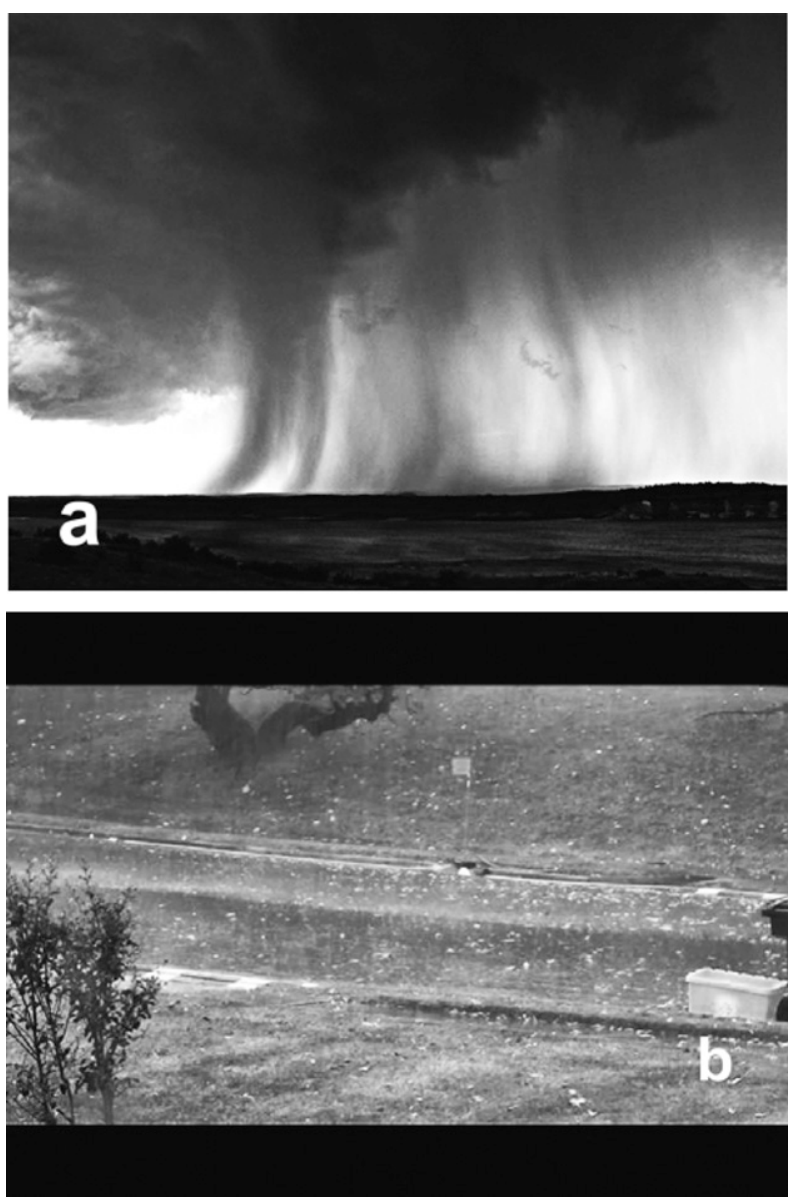

FIG. 1. (a) Picture of rain shafts illustrating the variability of the rain over many scales. The distance to the storm is several kilometers. (b) Example of a meter-scale white rain streak as it moved across a road delineated by the recycle bin in the lower right and the flag toward the upper center.

seconds while maintaining its overall structure. This alone highlights the potential difference between temporal and spatial clustering as well because a single instrument would have only seen the width of this streak in time as it passed over the instrument, and it would have entirely missed its length without additional instruments. It should come as no surprise, then, that spatial and temporal clustering likely differ.

This spatial heterogeneity seems to exist even on scales of centimeters (e.g., Jameson and Kostinski 2000, their Fig. 3 and text on p. 376; Kostinski et al. 2006) although that has not yet been directly measured. On the other hand, such bunching or clustering of the rain is also found up to at least the typical kilometer scales of most radar sample volumes (Jameson 2008). Thus, clustering appears to be an important characteristic of rain structure over a broad range of scales. What we do in this study is to look directly at some of the finest spatial scales from a few meters to $100 \mathrm{~m}$ directly rather than trying to infer them from temporal observations as was necessary in the past.

What do we mean by clustering? Specifically, clustering is the enhanced concentration (and dilution) of particles associated with increased (decreased) correlations of scatterers in neighboring volumes. That is, scatterers are said to be spatially correlated (usually expressed using the pair correlation function; e.g., Kostinski and Jameson 1997) when the number of drops occurring in an observation interval at one time or location is significantly correlated with the number of drops occurring at a different time or location. Expanded discussions can be found in Kostinski and Jameson (1997) and subsequent articles. On the other hand, statistical heterogeneity is the result of changing conditions such that the statistics of the observations depend upon the location or time of the measurements. Consequently, there is increased variance in rain observations 1) because of statistical fluctuations (often taken to be fluctuations in a Poisson distribution) are modulating "local" means (i.e., clustering; Jameson and Kostinski 1996, 1846-1848) and 2) because there are systematic changes in the observed longer-term or largerscale means associated with statistical heterogeneity.

Clustering can occur in both space and time. So far, however, all studies of clustering have only had access to temporal measurements. A spatial interpretation has then been inferred using an advection velocity to transform time into space. Features that advect unchanging past a detector are then simply linearly converted to their spatial equivalent. Until now, the validity of this assumption with regard to clustering remains unknown. Li et al. (2009) have already shown that such an assumption is not valid for the space-time covariance structure of a rain field on time and space scales greater than $15 \mathrm{~min}$ and $4 \mathrm{~km}$, respectively. One of the central purposes of this work is to investigate the likely acceptability of such a transformation on much finer scales with respect to individual drops.

A similar question arises with regard to DSD. That is, how representative is a temporally averaged DSD compared to a spatially averaged distribution? While a detailed response to this question is the subject of a different paper, using the network of instruments described below, we can at least provide some insight by considering the relation between the rainfall rate $R$ and the total number of drops $N_{t}$. In statistically homogeneous rain, it is known that $R \propto N_{t}$ exactly (Jameson and Kostinski, 2001b) where the constant of proportionality is related to a moment of the size distribution $P(D)$ and is therefore, a function of $P(D)$. Even in time series observations in statistically nonstationary rain, a similar 
relation but with much more scatter appears to hold (Jameson 2014) implying that an average $P(D)$ exists in statistically nonstationary rain. The question is, does it apply to spatial observations as well? We examine the answer below by considering differences between $R$ and $N_{t}$ for spatially averaged values over a network as compared to temporal time series relations for individual detectors. That is, if one were use the time series from a single detector and if one were to assume a simple advection velocity transformation for an estimate of $P(D)$ as expressed vis-á-vis a relation between $R$ and $N_{t}$, how much would that relation differ from that for spatially averaged values over a network?

One of the unique aspects of this study is the close proximity of so many optical disdrometers. To be sure, there have been other studies using networks of disdrometers but these have been focused more on different objectives. For example, data from a grid of six disdrometers separated by $1.25-1.4 \mathrm{~m}$ have been analyzed (Tokay et al. 2005) but only with respect to longterm averages. More recently, Tokay and Bashor (2010) considered measurements using three disdrometers along a line with a minimum separation of $600 \mathrm{~m}$. However, as in many past studies, that work focused on integrated quantities and parameters of an assumed form for the DSD rather than on the characterization of individual drop sizes. Most recently, Jaffrain and Berne (2012) report an important study in Lausanne, Switzerland. There they set up a network of optical disdrometers over a $1-\mathrm{km}^{2}$ area. Unlike our network, all but one $(85 \mathrm{~m})$ separation distances were greater than $100 \mathrm{~m}$. Moreover, in contrast with the work presented here, Jaffrain and Berne (2012) focused on spatial correlations of integrated quantities including the rainfall rate, the total number of drops, and the mass-weighted mean diameters. That is not our purpose here, where we are most interested in the small spatial and the temporal characterization at the level of individual drop sizes.

There are both practical and scientific reasons for wanting to understand better the meter-scale characteristics of rain. While such structures can affect the interpretation of radar measurements (Jameson 2008; Jameson and Kostinski 1996, 2008, 2010), for example, a more compelling practical concern is soil erosion. Nearly one-third of the world's arable land has been lost to soil erosion since 1950 (Pimentel et al. 1995). Moreover, soil erosion is estimated to have caused \$27.9 billion in the United States in 1997 alone (Uri and Lewis 1999). It is estimated by the USDA (http://www.nrcs. usda.gov/Internet/FSE_DOCUMENTS/nrcs142p2_ 051278.pdf) that $55 \%$ of all soil erosion is caused by water impacting the soil. This, of course, depends upon the kinetic energy of the drops (Kinnell 2005) and, therefore, it depends upon the drop size distribution. Most of this occurs as drops and clusters of drops dislodge the particles (e.g., Caracciolo et al. 2012), which are then carried away. A more complete understanding and parameterization of this process depends upon detailing the fine structures of the rain.

Aside from such practical justifications, however, there are also scientific reasons for having an interest in smallscale features in rain. For decades, the evolution of precipitation through collision, coalescence, and breakup has been considered to be adequately understood in time using one-dimensional models. Recently, however, it has become clear that the problem of precipitation evolution is, in time, truly three-dimensional (e.g., Jameson and Kostinski 2001a). That is, there is a spatial-temporal clustering or bunching of raindrops that complicates drop interactions more than can be handled using only a onedimensional model. Indeed, the very existence of rain streaks requires using greater spatial dimensionality. Since these interactions occur on scales of less than $1 \mathrm{~cm}$ up to several meters, depending on several factors such as the relative fall speeds of the interacting drops, it is important to begin to describe at least the two-dimensional spatial structures of rain. As of today, this has never been done on the appropriate spatial scales even at the ground much less aloft.

The central objective of this work, then, is to present some initial results from a unique network of 21 optical disdrometers that provide 1-min counts over 22 size bins to explore the differences between the spatial and temporal characterization of rain over dimensions less than $100 \mathrm{~m}$. In particular, with these spatial observations, we can finally compare spatial and temporal clustering (variability), and we can investigate the applicability of the advection velocity translation of temporal to spatial measurements used in past studies.

\section{A few definitions}

As explained in greater detail and as illustrated in the appendix, these disdrometers are spaced logarithmically along three arms extending from a common origin out to $100 \mathrm{~m}$ along two of the arms and out to $52 \mathrm{~m}$ on one arm that was limited by placement restrictions imposed by caretakers of the historical site. Two of the arms are orthogonal with the central one lying at $45^{\circ}$ between them. In addition, there is now a $2 \mathrm{D}$ video disdrometer placed near the origin that will be used to study microstructures but was not present for inclusion in this study. Thus, the focus here is on meter scales and times from minutes up to durations of the rain events. Before analyzing the data, however, we first describe some methodology and present a few useful definitions. 
In this work, there are both qualitative and quantitative observations. While the qualitative aspects will be readily apparent, it is necessary to briefly define two of the quantitative measures: namely, the temporal and the spatial pair correlation functions, which describe the clustering or bunching of objects either in time or in space. Consider a test volume $V$ containing $N=\bar{n} V$ drops. If we represent the random number of drops of a single size in a unit volume by $n$, say, then for a statistically homogeneous random field (in time and space), the joint probability $P(1,2)$ of finding two drops in small volumes $d V_{1}$ and $d V_{2}$ (one in each) separated by distance $l$ is given by (e.g., Green 1969, 62-63)

$$
P(1,2)=\bar{n}^{2} d V_{1} d V_{2}[1+\eta(l)],
$$

where $\eta(l)$ is the so-called pair correlation function. Note, however, that statistical homogeneity does not imply nor require physical homogeneity. Patchy, physically inhomogeneous rain can be fully consistent with statistical homogeneity. A complete derivation may also be found in Landau and Lifshitz (1980, p. 351). One of the original purposes of the pair correlation function was to quantify the scattering of light because of molecular clustering in liquids (Ornstein and Zernike 1914) with the onset of critical opalescence during phase transition. It has since been used to quantify the distribution of galaxies in space to determine whether they were distributed randomly (Peebles 1993,457-475) as well as the clustering of raindrops (Kostinski and Jameson 1997). While the pair correlation function can be applied to the total number of drops, in this work we largely focus on drops of a particular size.

More generally, the pair correlation function depends upon both time and location. This can be estimated operationally (Kostinski and Jameson 2000, p. 902) as

$$
\eta(\Delta l, \Delta t \mid D)=\frac{\langle n(l, t \mid D) \times n(l+\Delta l, t+\Delta t \mid D)\rangle-\mu(D)^{2}}{\mu(D)^{2}},
$$

where $n(l, t \mid D)$ and $n(l+\Delta l, t+\Delta t \mid D)$ are the numbers of drops given diameter $D$ in a unit volume at $(l, t)$ and $(l+\Delta l, t+\Delta t)$. Note that zero counts are valid contributions to $\eta$. When we let $l$ be fixed so that distance $\Delta l \rightarrow 0$, we have the pair correlation function in time. This is sometimes referred to as the clustering index (CI) (e.g., Baker and Lawson 2010; Chaumat and Brenguier 2001; Larsen 2012). Equation (2) then becomes

$\eta(0, \Delta t \mid D)=\frac{\langle n(l, t \mid D) \times n(l, t+\Delta t \mid D)\rangle-\mu(D)^{2}}{\mu(D)^{2}}$.
That is, in practice, using one instrument we count the number of drops every unit interval, in this case $1 \mathrm{~min}$, over each size bin over the observation interval $T$. This is our time series of observations. For each time lag $\Delta t$ (in this case an integer number of minutes) and for each drop size bin, we then compute the average value of $n(l, t) \times n(l, t+\Delta t)$ over $T$ (denoted by the angle brackets). We then divide this result by the square of $\mu^{2}$, the average number of drops every unit interval computed for that instrument over $T$, and subtract 1 . In the limit as $\Delta t \rightarrow 0$, we then have the clustering index coefficient (CIC) as discussed in many papers (e.g., Jameson and Kostinski 1999, p. 3924). It provides a measure of the strength of the clustering of the drops with respect to time.

In an analogous manner, operationally the spatial pair correlation function (SPCF) along a line is simply given setting $\Delta t$ to 0 ; that is,

$\eta(\Delta l, 0 \mid D)=\frac{\langle n(l, t \mid D) \times n(l+\Delta l, t \mid D)\rangle-\mu(D)^{2}}{\mu(D)^{2}}$.

In practice, we count the number of drops every minute over each size bin over interval $T$ for two instruments separated by $\Delta l$. This is our time series of observed SPCF. For each $\Delta l$ and for each drop size bin, we then compute the average value of $n(l, t) \times n(l+\Delta l, t)$ over $T$. We then divide this result by the mean value squared and subtract 1 . In the limit as $\Delta l \rightarrow 0$, we then have the spatial pair correlation function coefficient (SPCFC).

To maximize the number of different $l$ values, and, therefore, to provide a more complete estimate of SPCF, the instruments do not necessarily lie along the same line. This, in turn, means that different orientations contribute to SPCF so that the noise will be enhanced depending on the amount of anisotropy if it exists. That is, (4) is defined for observations along a single line. Each line has a direction. Conditions along different lines may be different if there is anisotropy. Combining observations along different lines then contributes to the variability in the observed values increasing noise around a mean SPCF. We will use SPCFC to denote the coefficient of the spatial pair correlation function when $l=0$. Note that in general SPCFC will not equal the CIC because sampling in time and space are not necessarily equivalent. Unlike SPCF, SPCFC is a measurement at a point so that it will not be affected by any anisotropy should it exist.

A comparison among the outputs for all the instruments over the entire interval showed no consistent biases. That is, the instruments measure counts. The counts from any one instrument were never consistently nor persistently too high nor too low at any drop size. For example, sometimes one instrument would have an 
abundance of small drops, but the next time, a different instrument would have a surfeit of small drops. Furthermore, for the two time periods identified below, the record counting statistics approach of Anderson and Kostinski (2010) over the most relevant drop counts (0.6-4 mm) showed that over each observation interval separately the data were statistically stationary to a high degree of reliability (within $1 / 4-1 / 2 \sigma$ bounds). With respect to the spatial pair correlation, there are $171 \mathrm{com}$ binations of separations over $440 \mathrm{~min}$ of observations and 22 drop bins (although we restricted analyses to $0.625-6.25 \mathrm{~mm}$ ). With respect to CIC, there are 440 observations per instrument for calculations. These are more than sufficient for accurate estimates of both CIC and SPCFC. Nevertheless, we are dealing with finite samples so that the bracketed quantities in (2)-(4) are subject to sampling uncertainties. These are estimated next.

Specifically, the relative error of $z=\sigma^{2} / \mu^{2}$ is the sum of the relative errors of the sample mean squared and of the sample variance, which goes as $K / N-\sigma^{4} \sqrt{[(N-3) / N(N-1)]}$ (e.g., Mood et al. 1974), where $\mu$ is the mean counts per minute per instrument over the interval, $N$ is the sample size (number of minutes in this case), and $K$ is the kurtosis of the distribution of observed $n$. Many distributions of drop counts $n$ are represented by gamma distributions for which the kurtosis is $K=3+6 z$. The variance of the sampled mean squared, however, goes as $2 \sigma / \sqrt{N}$. Putting this all together, we have

$$
\frac{\Delta z}{z}=\frac{1}{\sqrt{M}} \frac{1}{\sqrt{N}}\left[\frac{2 \sqrt{z}}{\mu}+\sqrt{\left|\left(\frac{3}{\mu^{4} z^{2}}+\frac{6}{\mu^{4} z}-\frac{N-3}{N-1}\right)\right|},\right.
$$

where $M$ is defined below. For the observations in this work, when $\mu \leq 5$, we still have $z \geq 2$ and when $\mu \geq 10$, we find $\mathrm{z} \geq 0.3$ so that with rare exceptions we can ignore the $\mu^{-4}$ terms; then (5) simplifies to

$$
\frac{\Delta z}{z} \cong \frac{1}{\sqrt{M}} \frac{1}{\sqrt{N}}\left[\sqrt{\frac{(N-3)}{N-1}}+\frac{2}{\mu} \sqrt{z}\right]
$$

where $z$ is either CIC or SPCFC when $M$ is unity. This is used for the uncertainty calculations illustrated later. We also note that (6) applies not just to gamma distributions but to all symmetric distributions including the normal distribution. For CIC and SPCFC, $M$ is unity while, for SPCF, $M$ is the number of pairs of instruments contributing to the estimate SPCF at separation $l$.

With these tools we begin the analyses of a rain event consisting of both a convective and a stratiform component

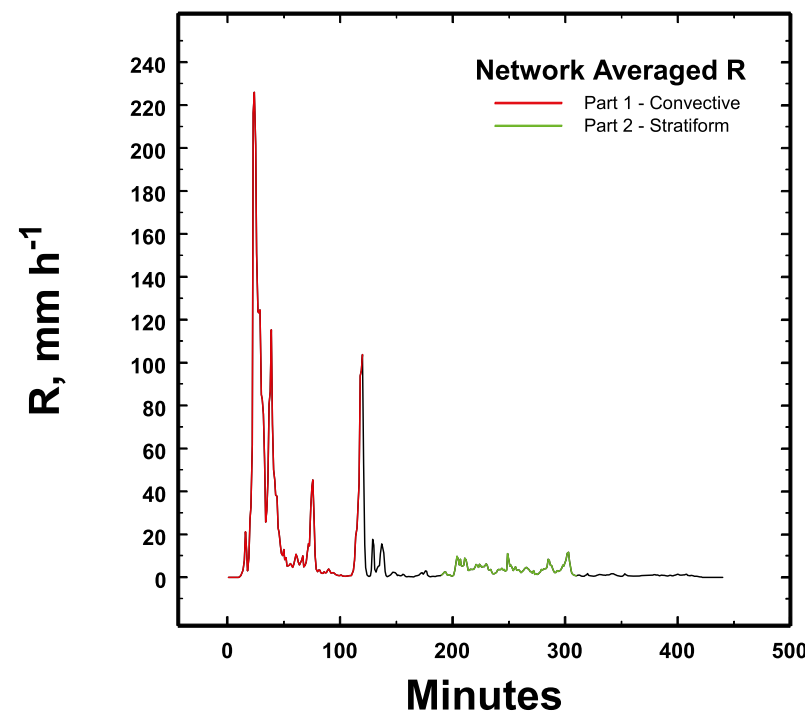

FIG. 2. Network-averaged rainfall rate during the 440-min event. The colors denote the two analyzed periods each $120 \mathrm{~min}$ in length.

that began at 1645:00 UTC 23 November 2013 (Saturday) and lasted for $440 \mathrm{~min}$ with maximum rainfall rates once approaching $250 \mathrm{~mm} \mathrm{~h}^{-1}$.

\section{Analyses}

\section{a. First example}

To get a basic feeling for this rain event, the rainfall rate averaged over the entire network is illustrated in Fig. 2. Only 2 of the 21 detectors were not in operation during this event (detectors $\mathrm{L}$ and $\mathrm{M}$ as shown in the appendix) so that this average is over 19 of the optical disdrometers. The two selected time periods for this part of the analyses are as indicated. Each is 120 min long.

Because of the obvious differences in the rainfall rates, it should come as no surprise that there will be differences in the plots of the total accumulated rainfalls during each period. In Fig. 3 we plot the total rainfall within $30 \mathrm{~m}$ of the network origin. This highlights the appearance of structures even over areas as small as $900 \mathrm{~m}^{2}$. Figure $3 \mathrm{a}$ is for the convective rain, while Fig. $3 \mathrm{~b}$ is for the stratiform precipitation. The convective rain produced a variation (largest minus smallest amounts) of $18 \mathrm{~mm}$ ( $34 \%$ of the area mean) in the accumulated rainfall over $2 \mathrm{~h}$ over $900 \mathrm{~m}^{2}$. This compares to the $5 \mathrm{~mm}$ in accumulated rainfall over $2 \mathrm{~h}$ for the tipping-bucket measurements of Larsen et al. (2010). In contrast, the stratiform accumulated rainfall was obviously much more spatially uniform. Here the difference between the maximum and minimum amount was only $2 \mathrm{~mm}(27 \%$ of the area mean) over $2 \mathrm{~h}$. 


\section{Total Rainfall $120 \mathrm{~min}, \mathrm{~mm}$}

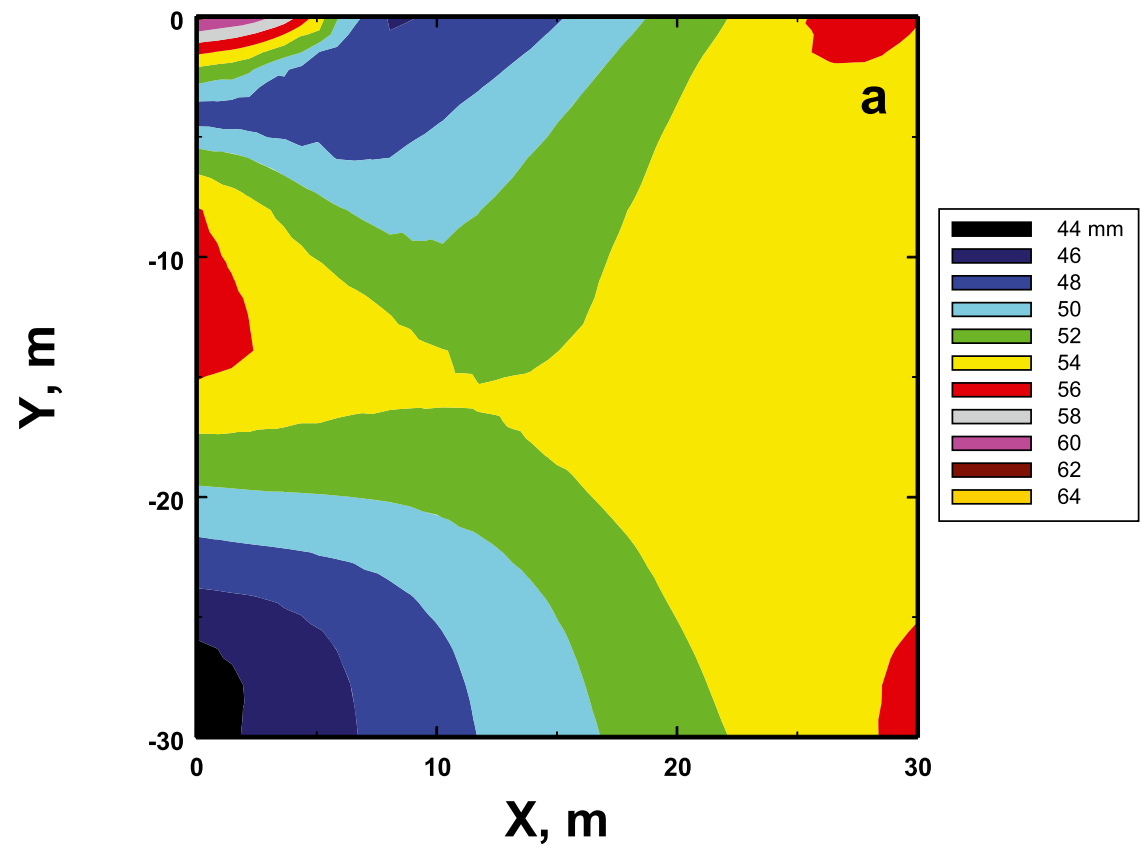

\section{Total Rainfall 190-310 min, mm}

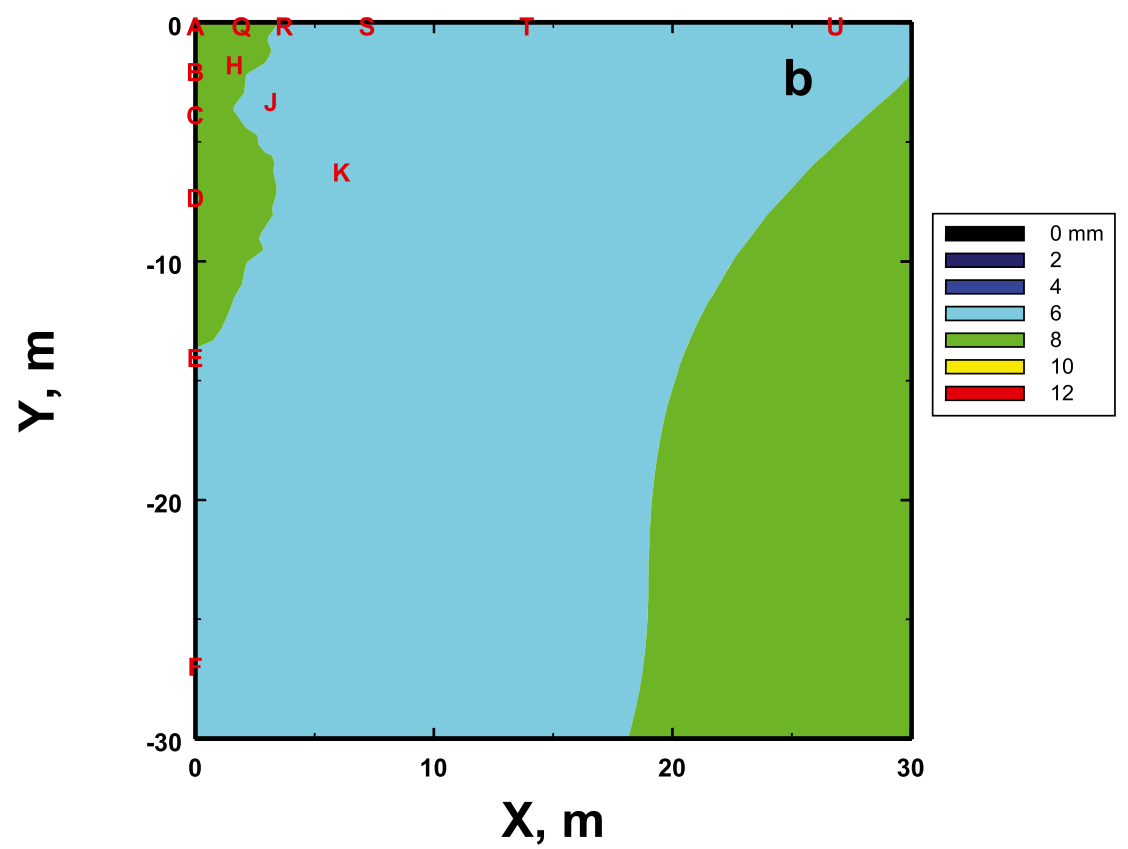

FIG. 3. Total rainfall for (a) the more convective and (b) the more stratiform rain as discussed in the text. The letters in (b) refer to the optical disdrometer identifiers within $42 \mathrm{~m}$ of the origin. Note, however, that the contours are based on the observations by all the instruments interpolated over $100 \mathrm{~m}^{2}$ using the conservative method of Watson (1994).

One of the most important physical origins of the variability of the rainfall is drop clustering (Jameson and Kostinski 1999, 2002; Jameson 2008). While Fig. 3 might then seem to imply that drop clustering is greater in the convective than in the stratiform rain, this question must be addressed carefully. First, drop clustering is a function of drop size so that we must know which sizes are contributing the most to each type of rain. We do this by 


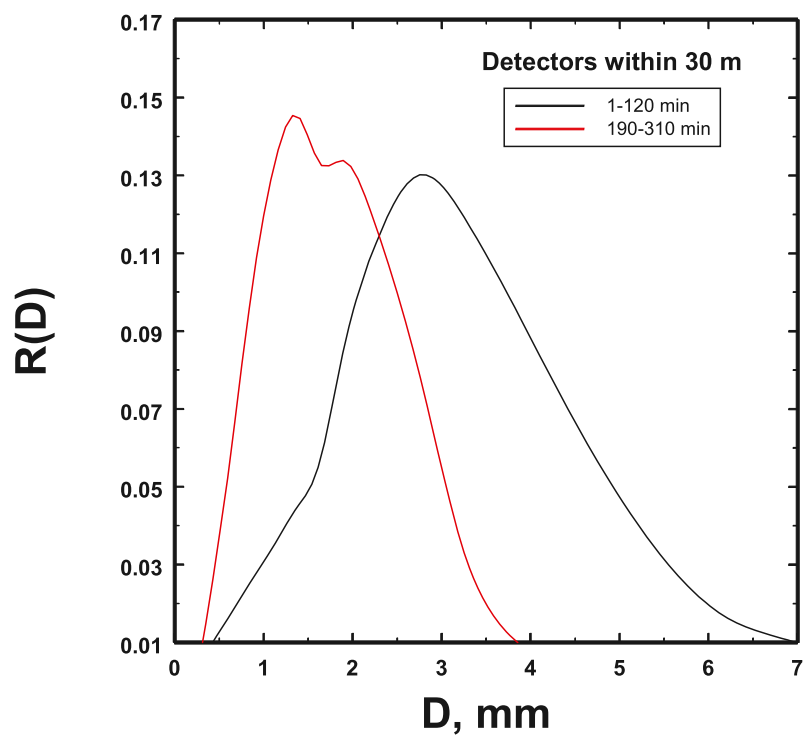

FIG. 4. The fractional contribution to the rainfall rate by the different drop diameters in the more convective rain (black) and the more stratiform rain (red) for detectors within $30 \mathrm{~m}$ of disdrometer A.

first looking at the fractional contribution to the rainfall rates for different drop sizes for both the convective and stratiform rain over their respective intervals as shown in Fig. 4. As conventional wisdom would suggest, the contribution to the convective rain (black line) comes mostly from the large drops, in this case peaked around 3-mm diameter. On the other hand, the stratiform lighter rain (red line) is derived mostly from drops in the 1-2-mm-diameter sizes.

With this perspective from Fig. 4, we now look at the temporal clustering index (CIC) as a function of drop size for both types of rain. For convenience only the detectors along arm 3 (see appendix) are illustrated in Fig. 5 , but calculations show exactly the same results for the other arms as well.

The forms of the two plots are quite similar with the exception that, in the convective rain, the peak is near $1-\mathrm{mm}$ diameter whereas it is somewhat smaller in the stratiform rain. However, there are two significant features to notice. First, CIC in the stratiform rain is 10 times larger than that in the convective rain. This suggests that the clustering in time is more important in the stratiform rain than in the convective rain. Second, CIC at $3-\mathrm{mm}$ diameter is small in the convective rain, suggesting that temporal clustering is not important. These observations imply that temporal clustering of the rain should be small in the convective case and that it should be much greater in the stratiform than in the convective rain. So where does the clustering apparent in the variable rain rate come from in the convective rain?
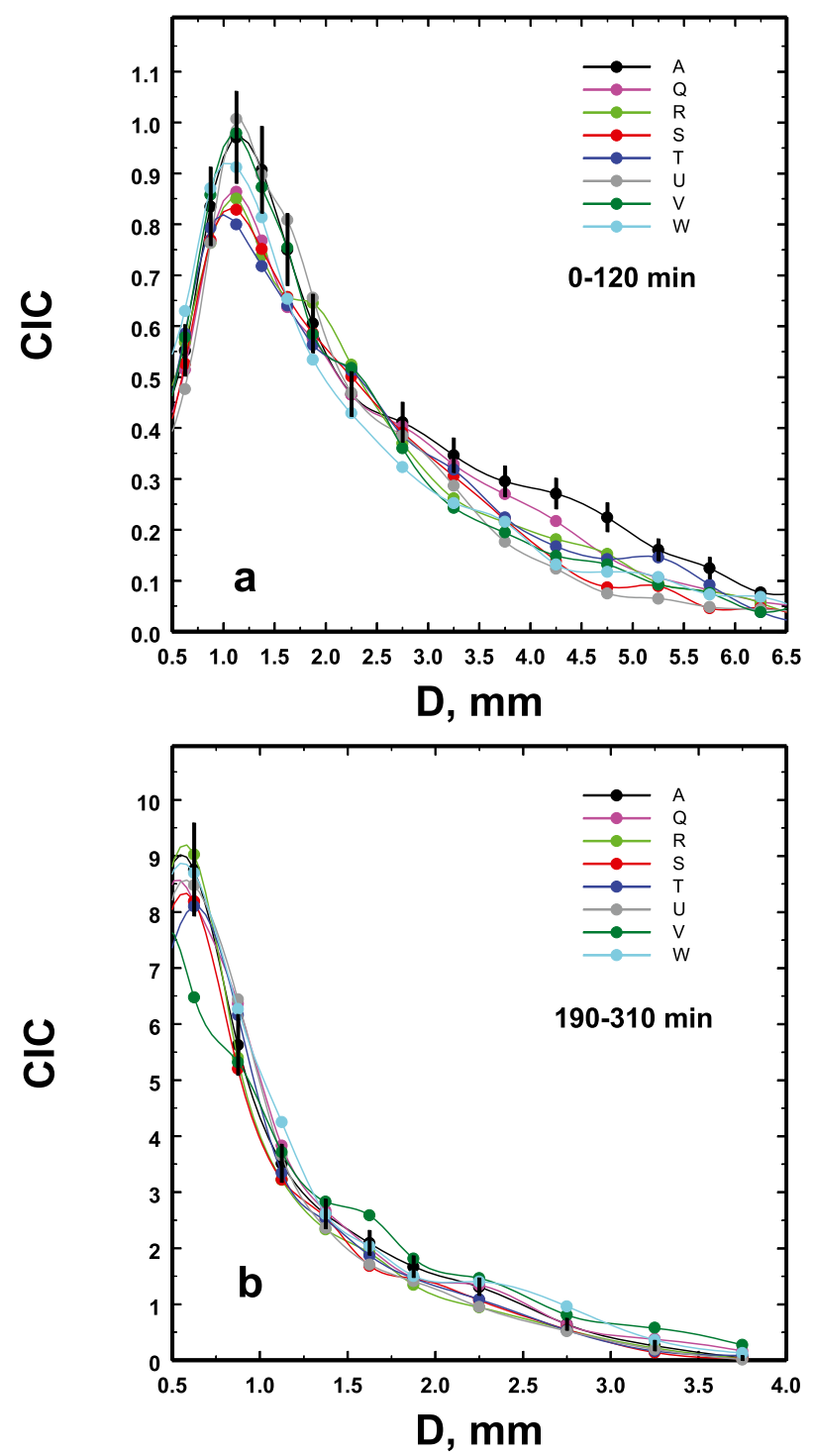

FIG. 5. CIC along arm 3 in (a) the more convective and (b) the more stratiform rain. CIC is obviously much larger in the stratiform rain than in the convective rain, which is counterintuitive to the idea that clustering should be larger in the much-more-variable convective rain. The letters refer to the particular detector (see appendix). The vertical bars indicate $\pm 1 \sigma$ uncertainties for detector A mostly owing to sample size.

This can be addressed by considering the spatial pair correlation functions (SPCF) as shown in Fig. 6. The computations involved pairs having different spatial orientations. This likely produces some of the scatter in Fig. 6, although recently Jaffrain and Berne (2012) argued that such paired measurements tend toward isotropy even on sub-1-km scales. In the convective rain (Fig. 6a), we now have values that are several times larger than in the stratiform rain, which is consistent with the observed patchiness of the rainfall. In the 

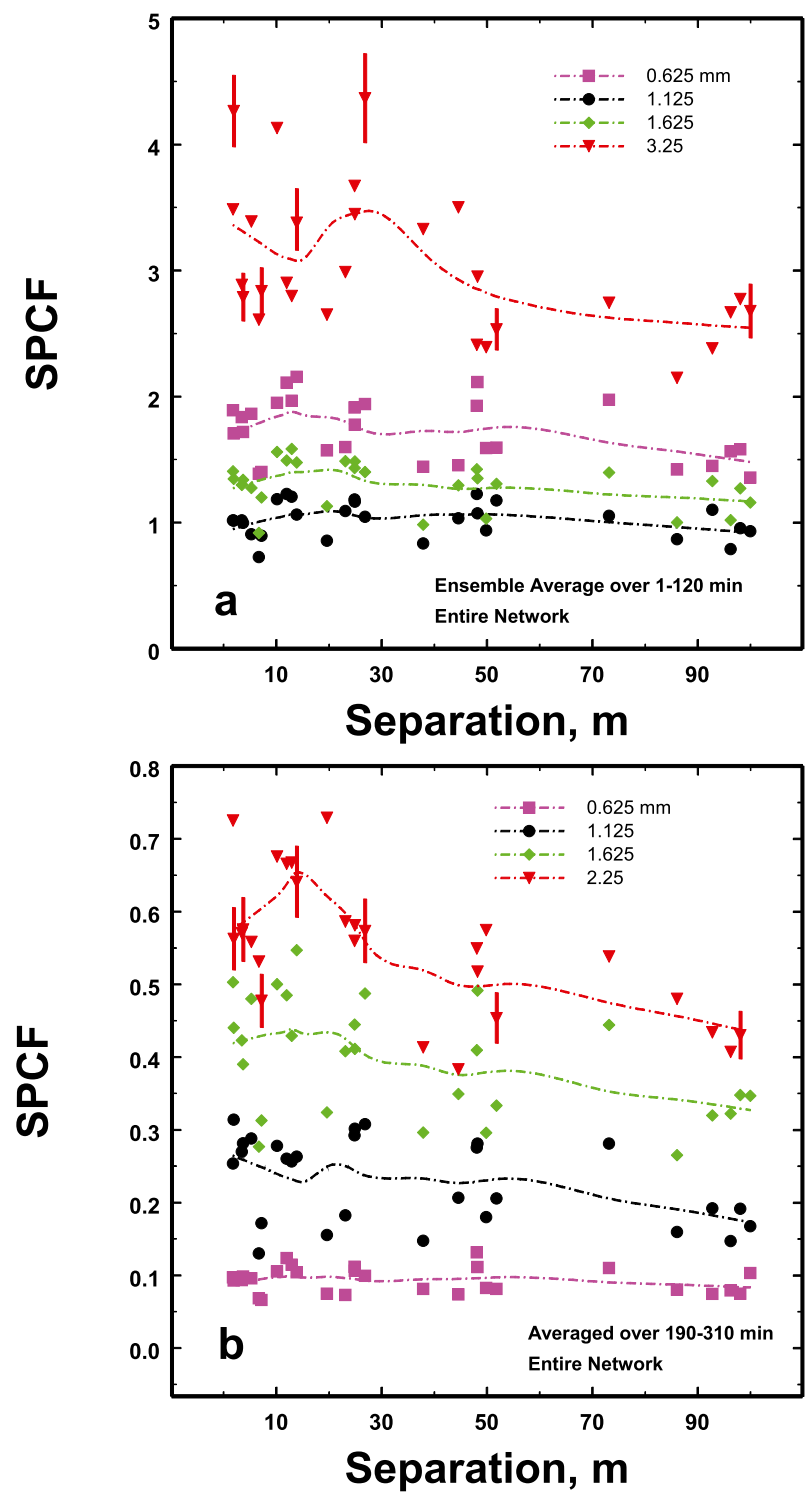

FIG. 6. Plots of the spatial pair cross-correlation function for five different drop sizes in (a) the more convective and (b) the more stratiform rain as discussed in the text. Fluctuations around the mean curve approximately indicate the level of uncertainty. Some $\pm 1 \sigma$ uncertainty bars at a few selected separations have been added for the largest drop sizes to give a feel for some of the largest sampling uncertainties.

convective rain, CIC and SPCF agree for the 1-mm drop size so that at that size, the spatial clustering and the temporal clustering contribute about equally. However, at the larger drop sizes that contribute most to the rainfall rate in the convective rain, significant clustering is evident in SPCF but not in CIC, implying that for those drops, spatial clustering dominates over weak temporal clustering. In the stratiform rain, however, the SPCF values (Fig. 6b) are all much smaller than the CIC

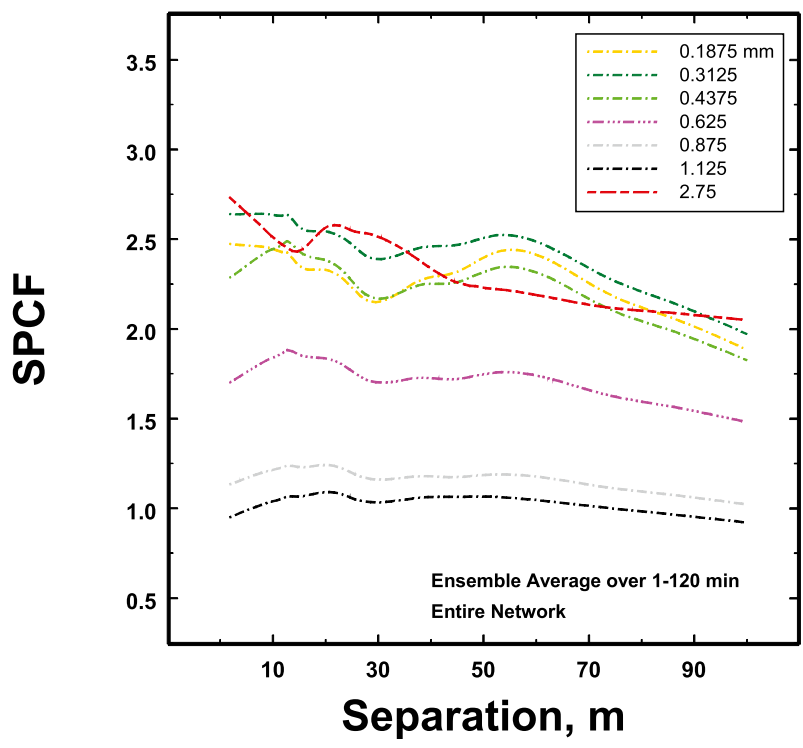

FIG. 7. The spatial pair cross-correlation functions at the indicated sizes for the convective rain over 1-120 min. The coincidence of the smallest sizes with the larger size suggests a possible role of drop breakup in the generation of the smaller drops as discussed in the text.

values, particularly at the most important drop sizes of $1-2 \mathrm{~mm}$, suggesting that the rain is dominated by temporal, not spatial, clustering.

There are also a few other features worth mentioning. One is that there are hints of a gradual decrease in SPCF with increasing separation distance in both the convective and the stratiform rain as one would expect. Second, whereas there is a monotonic increase in the SPCF with increasing drop size in the stratiform rain, this does not happen in the convective rain. Specifically, in Fig. 6a, SPCF is greater at $0.625-\mathrm{mm}$ diameters than at 1.125 and $1.625 \mathrm{~mm}$. This is highlighted in Fig. 7, where the SPCF values in the convective rain are plotted for all the small drop sizes less than $0.625-\mathrm{mm}$ diameter and for the larger drops. It is particularly interesting that the SPCF values are so similar for both the smallest and largest drop sizes that contribute most to the rainfall rate, yet at intermediate sizes, the SPCF values appear to behave independently.

There are at least two possible explanations. One is that this is some kind of instrument effect. This seems unlikely since there are plenty of drops at all these sizes and since the behaviors at all of the smaller sizes appear to be consistent. An alternative, but unproven, explanation could be that in the convective, intense rain, the spatial pair correlation (SPCF) of the larger drops is conserved as the small drops are created during drop breakup of the large drops. This is consistent with recent observations of superterminal fall speeds of small drops 

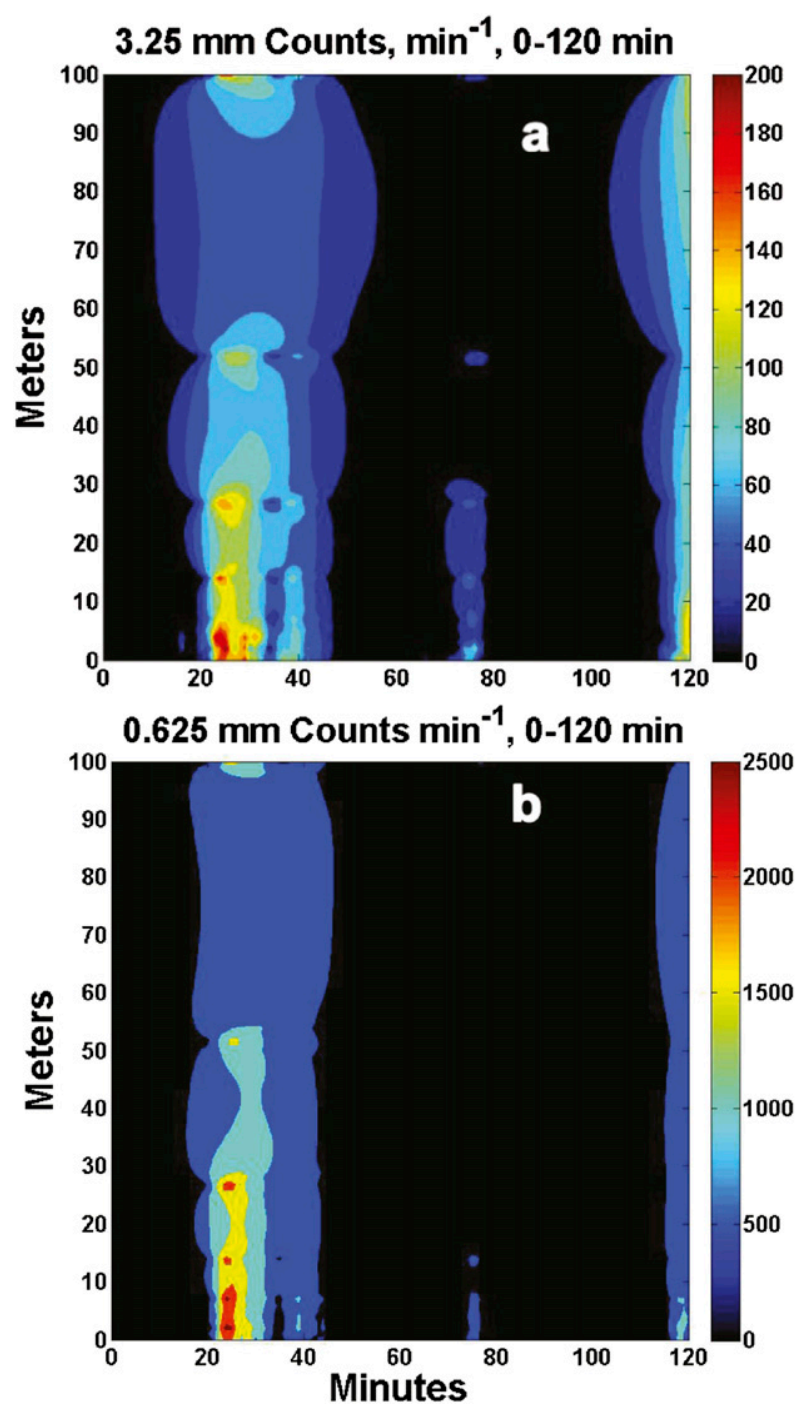

FIG. 8. Contour plots of the counts at a large and a small drop size as a function of time and spatial separation along arm 3 suggesting that the spatial variability dominates at both sizes as discussed in the text.

due to the recent breakup of large drops (MonteroMartínez et al. 2009; Larsen et al. 2014).

To explore further, the number of counts per minute are plotted as a function of time and separation distance for the convective rain for drop sizes of 3.25- (Fig. 8a) and $0.625-\mathrm{mm}$ diameters (Fig. $8 \mathrm{~b}$ ). In both cases where rain exists, spatial variability (clustering) dominates (also as implied by SPCF), not temporal variability (as also reflected in the small values of CIC). That is, one picks a time - say, $22 \mathrm{~min}$-where most of the variability is in the vertical direction (spatial) with less variability between 20 and $40 \mathrm{~min}$ in neighboring times (horizontal). Moreover, there is a considerable degree of overlap of the features in these two plots so that where there are
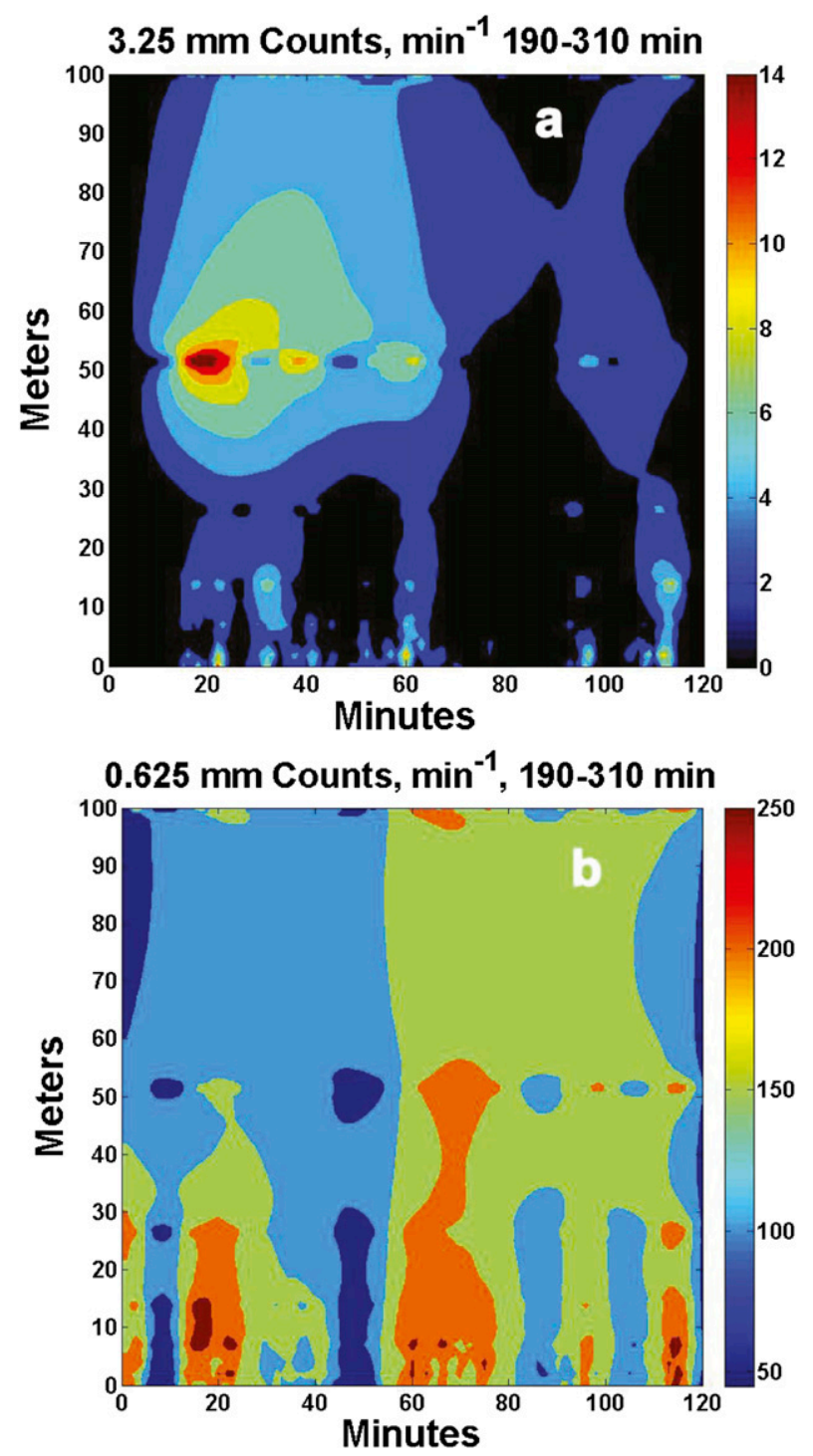

FIG. 9. Contour plots of the counts at a large and a small drop size as a function of time and spatial separation along arm 3 , suggesting that the large size is dominated by spatial variability while the small size is dominated by temporal variability as discussed in the text.

maxima in the large drops, there tend to be maxima in the small drops, for example. In fact, the normalized 2D cross-correlation coefficient (Haralick and Shapiro 1992, 316-317) between the two data fields is 0.964 . While this does not prove that many of the small drops are created by the breakup of larger drops, these features strongly contrast with the stratiform data as illustrated in Fig. 9.

Although the variability for the large drops persists (likely because embedded pockets of convective rain passed through the stratiform rain), the opposite is true for the smaller drops (Fig. 9b) for which the variability is largely but not exclusively more along the temporal 


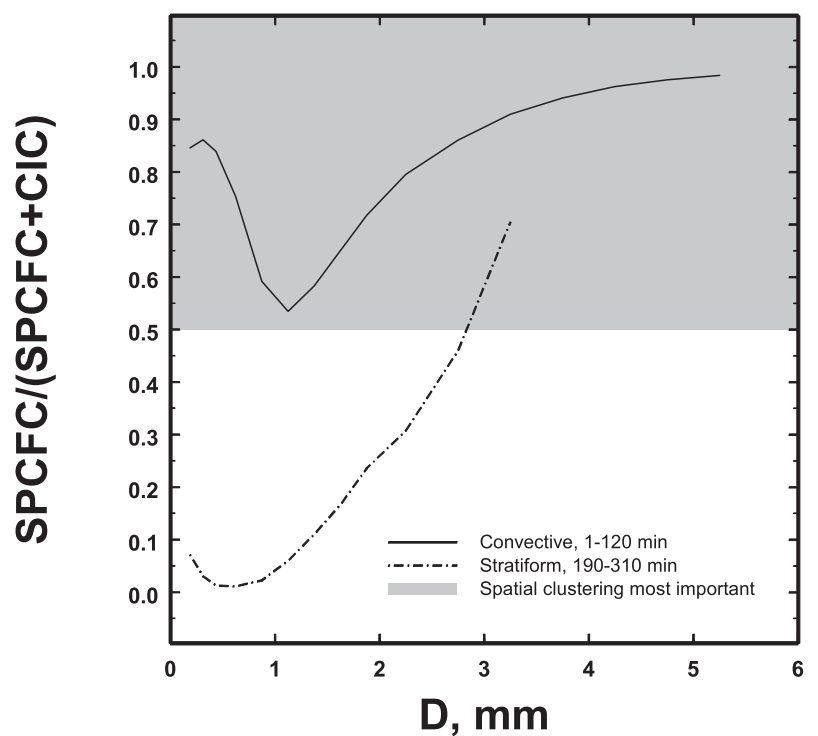

FIG. 10. Plots of the fractional contribution that the spatial clustering makes to the sum of spatial and temporal clustering indices in the more convective rain (solid) and the more stratiform rain (dotted-dashed) as discussed further in the text.

direction. Consequently, the normalized 2D crosscorrelation coefficient between the two data fields (Figs. 9a and 9b) is only about -0.106 . That is, the two data fields are statistically independent in the case of stratiform rain because of the different roles played by spatial and temporal clustering at these two drop sizes.

To look at all the different drop sizes, the fractional contributions of SPCFC to the sum of SPCFC and CIC (both averaged over the same detectors) are plotted in Fig. 10 as a function of drop size for the two sets of data. Clearly, at all drop sizes, the spatial clustering predominates in the convective rain while it only predominates at the very largest sizes in the more stratiform case. This latter observation and the fact that such large drops are found at all probably suggests that small convective elements occasionally passed through the background of stratiform rain.

These two sets of observations illustrate the differences between the relevance of CIC and of SPCF for the different drop sizes in different kinds of rain. For the large drops the variability largely remains spatial so that SPCFC more accurately reflects the relevant clustering; or, to put it another way, large drops tend to come more in spatial bunches than in temporal bunches. On the other hand, for the small drops, temporal variations dominate in the stratiform rain so that spatial variability is not as important; that is, the clustering is more in time than in space (Fig. 9b). The important point is that in general, an adequate description of drop clustering and

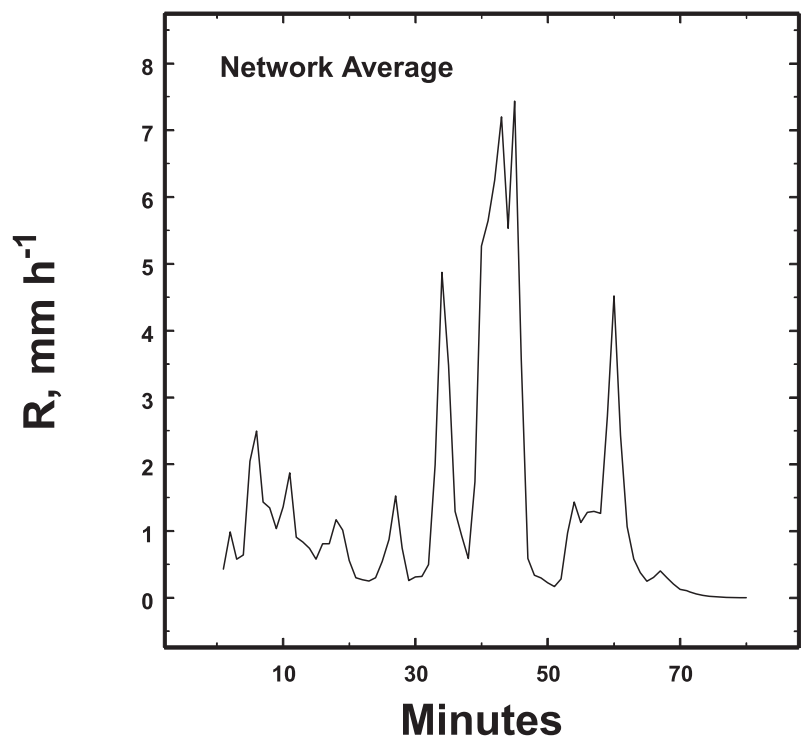

FIG. 11. Time profile of the network-average rainfall rate.

rain patches requires not just temporal observations but also spatial observations and one is not equivalent to the other. A simple advection velocity transformation between the spatial and temporal variability (clustering) seems highly unlikely particularly over all the different drop sizes.

These examples show that even simple rain events can possess complex characteristics. One anticipates, then, that most natural rain will consist of these two components to varying degrees at different times and locations. This is illustrated next.

\section{b. Second example}

This is the analysis of a case of light rain with the occasional embedded heavier rain collected beginning at 1747 UTC 26 December 2013. This example represents a mixture of the two previous cases. As the rain-rate profile indicates (Fig. 11), there was a background of light rain (around $1 \mathrm{~mm} \mathrm{~h}^{-1}$ ) with a few short bursts of heavier rain of up to $7 \mathrm{~mm} \mathrm{~h}^{-1}$. The effect of these short bursts is reflected in the total accumulated rainfall over the 80-min rain event (Fig. 12), where the field is very uniform with the exception of a local maximum in the upper-left corner. Only the closest $30 \mathrm{~m}$ from detector A are shown to highlight what structure was evident in an otherwise very uniform field. Nevertheless, the difference between the maximum and the minimum total rainfall, while only $0.6 \mathrm{~mm}$, is still $34 \%$ of the mean value.

An inspection of the fractional contribution to the rainfall rate (Fig. 13) with respect to drop size reveals three peaks. The first two are less than and approaching 


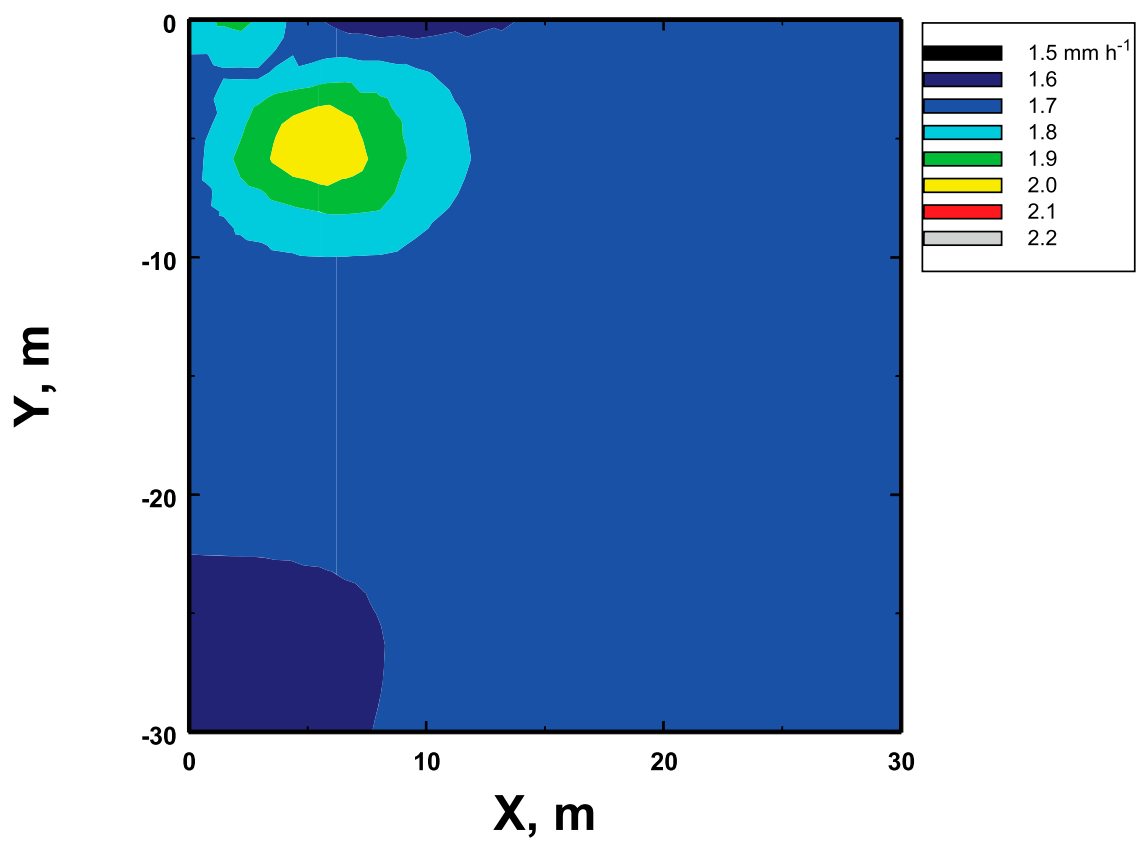

FIG. 12. Total accumulated rainfall in the closest $30 \mathrm{~m}$ to the origin.

1-mm diameter, respectively, accounting for a little more than $80 \%$ of the rainfall rate. The third is found around $2.25 \mathrm{~mm}$ with drops larger than $2 \mathrm{~mm}$ accounting for only $10 \%$ of the rainfall rate. This certainly suggests a combination of stratiform (a lot of smaller drops) that account for most of the rain rate and some convective rain (large drops), which appear to account for about $10 \%$ of the rain rate.

A comparison between CIC (Fig. 14a) and SPCF (Fig. 14b) shows that the small drops are clustered in

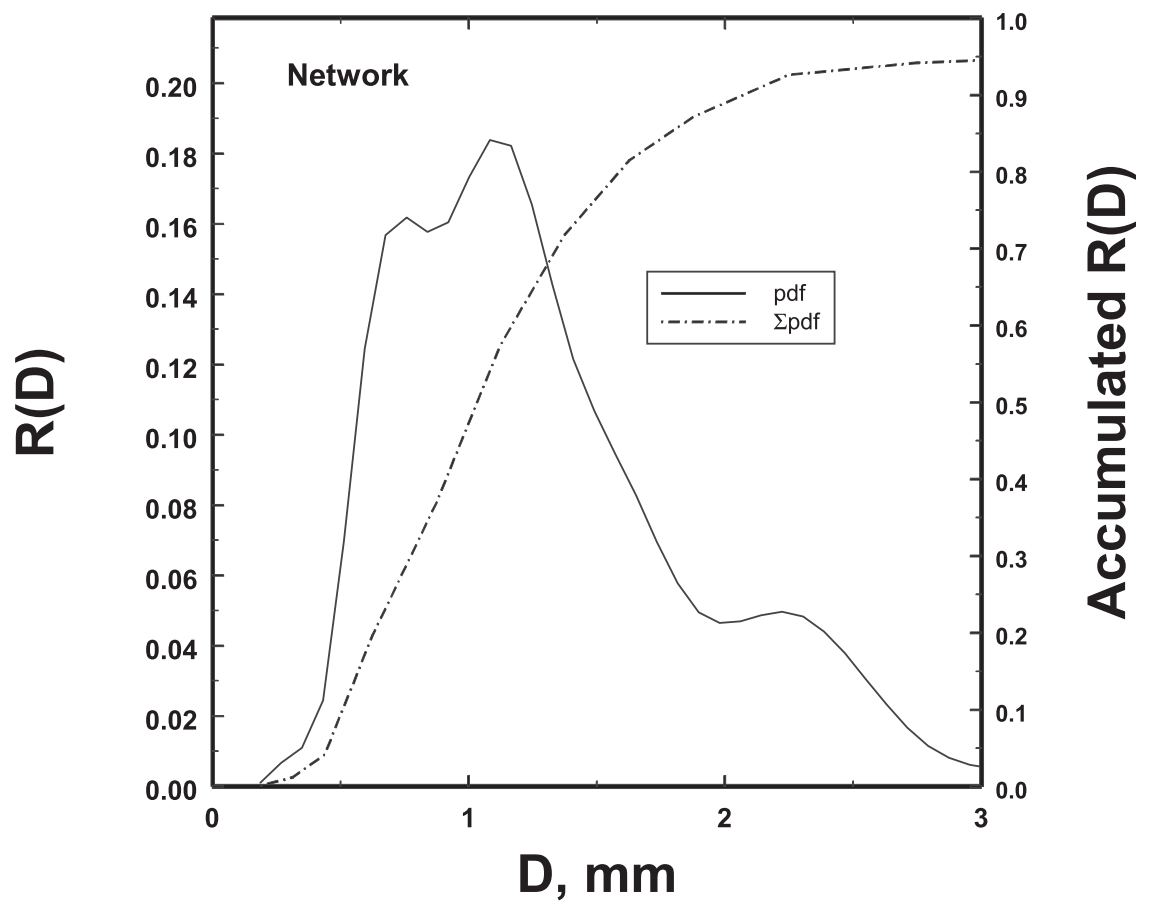

FIG. 13. The fractional contribution to the rainfall rate by the different drop diameters and its accumulated value ( $\Sigma$ pdf) for detectors within $30 \mathrm{~m}$ of disdrometer A. 

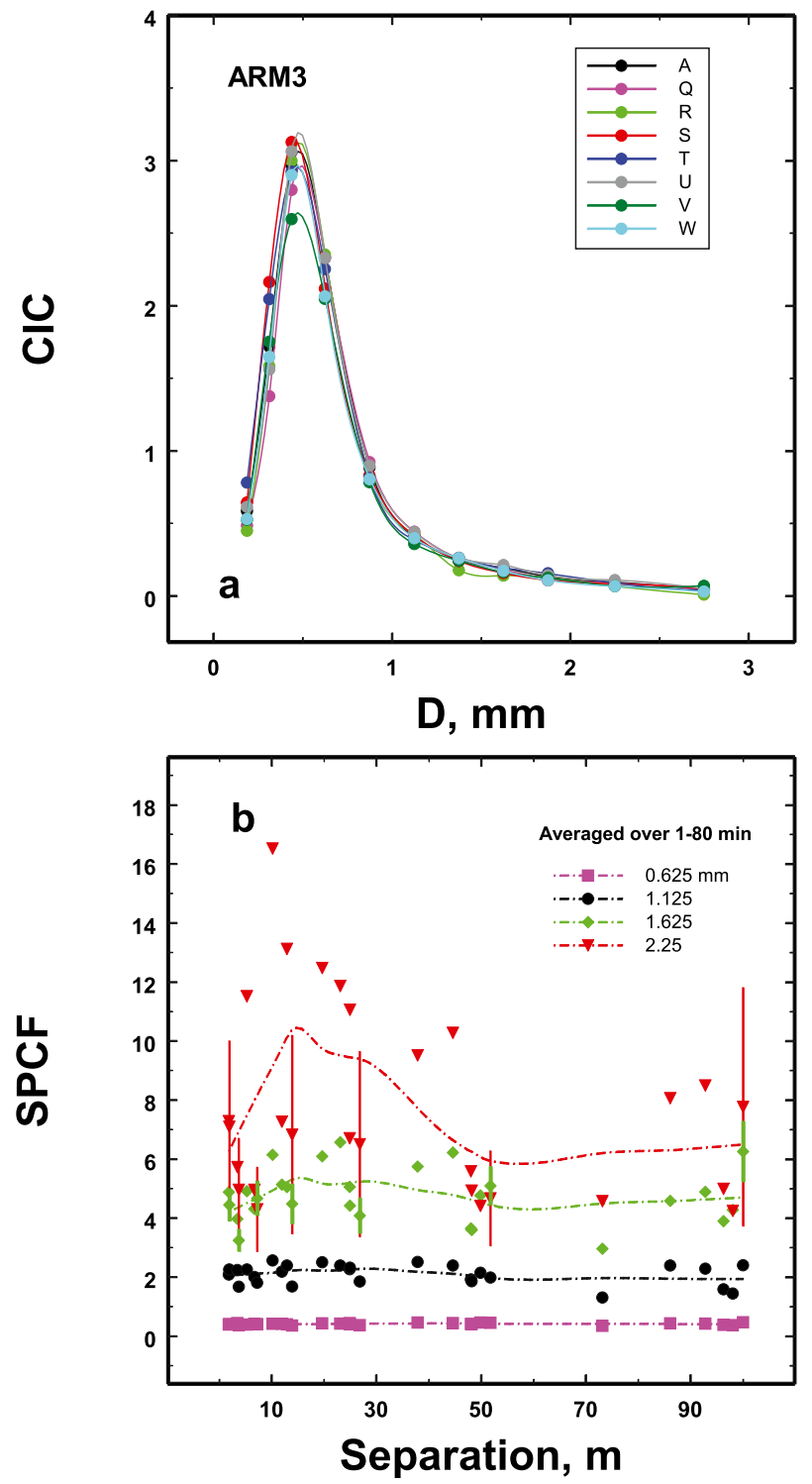

FIG. 14. Plots of (a) CIC and (b) SPCF for these data. Fluctuations around the mean curve approximately indicate the level of uncertainty. Some $\pm 1 \sigma$ uncertainty bars at a few selected separations have been added for the largest drop sizes to give a feel for some of the largest sampling uncertainties. At the largest sizes, the uncertainty is greater than in Fig. 6 because of the shorter sample period and greater scarcity of the largest drops ( $\mu$ near unity). Note that in (b) as the average number of drop counts per minute per instrument decreases from an average of $4 \mathrm{~min}^{-1}(1.625 \mathrm{~mm})$ down to $1 \mathrm{~min}^{-1}(2.25 \mathrm{~mm})$, the sampling uncertainty increases substantially.

time while the larger drops are clustered in space. Specifically, with the exception of the smallest drop size bin, Fig. 15 shows that temporal clustering dominates for sizes less than about $0.8 \mathrm{~mm}$. For diameters greater than $1 \mathrm{~mm}$, the spatial clustering dominates by at least 2:1. As before, $\mathrm{CIC}$ and SPCFC are averaged over the same detectors.

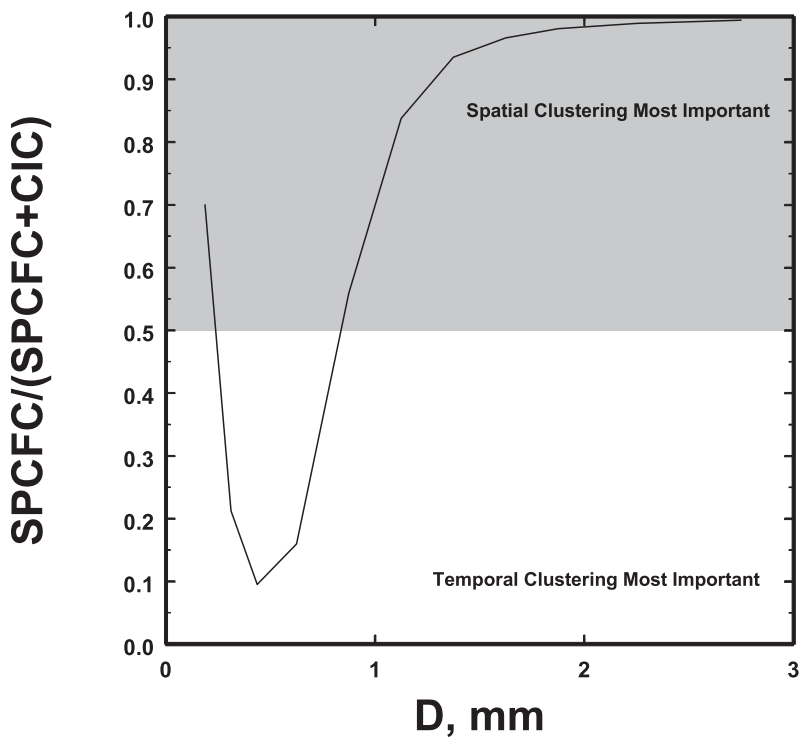

FIG. 15. Fractional contribution of the spatial clustering to the sum of spatial and temporal clustering indices as a function of drop size.

It is not surprising, then, that the plots of SPCF in time and separation distance are also different between the small and large sizes as illustrated in Fig. 16. This particular rain event apparently consisted of a precipitation event of small drops mostly correlated in time and another component of larger drops mostly correlated in space. This is, then, more like a combination of the two previous examples also reflected in the normalized 2D cross-correlation coefficient of 0.396 between Figs. 16a and 16b. This value is larger than in the more stratiform case, but it is smaller than in the more convective rain analyzed previously. The structure of rainfall, therefore, can best be understood by simultaneously considering both the temporal and the spatial correlation functions, which, as we have seen, also depend upon the sizes of the drops.

Aside from these conclusions, differences between the spatial and temporal characteristics show up in other ways as well, as discussed next.

\section{c. On the relation between $R$ and $N_{t}$}

As discussed previously, in statistically homogeneous rain, it is known that $R \propto N_{t}$ because the expected value of $R$ is exactly proportional to the expected value of $N_{t}$ times the expected value of $D^{3} V_{t}$ (Jameson and Kostinski 2001b; Steiner et al. 2004), where $V_{t}(D)$ is the terminal fall speed of drop of diameter $D$. Since $V_{t}(D)$ is a function of drop diameter, the expected value of $D^{3} V_{t}$ obviously depends on the frequency distribution of drop sizes $P(D)$, so, in that sense, this relation is a weighted 

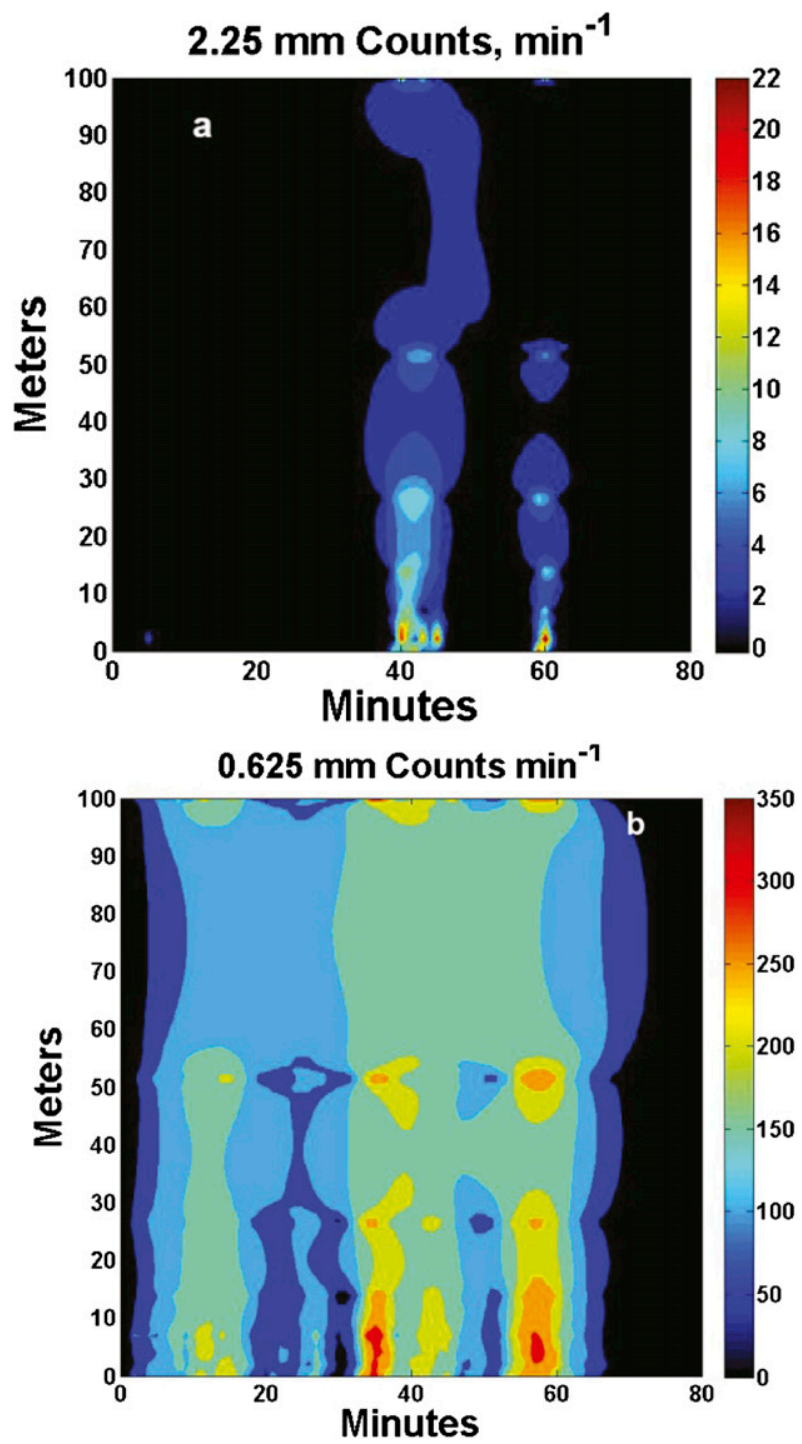

FIG. 16. Separation-time contour plots for (a) the large drop and (b) the small drop counts as discussed in the text.

proxy for $P(D)$. For statistically heterogeneous rain, however, there is no theoretical reason to expect a linear relation between $R$ and $N_{t}$ because $P(D)$ potentially changes with each new observation until the measurements cease. However, in previous work, Jameson (2014) demonstrated using one disdrometer that even in statistically heterogeneous conditions, temporal measurements of the rainfall rate and the total number of drops in a unit volume $N_{t}$ are highly correlated and essentially linearly related. By contrast, $R$ is poorly correlated with a different characteristic parameter of $P(D)$-namely the mean drop size (the inverse of the slope of an exponential size distribution). These same results appear here in the 19

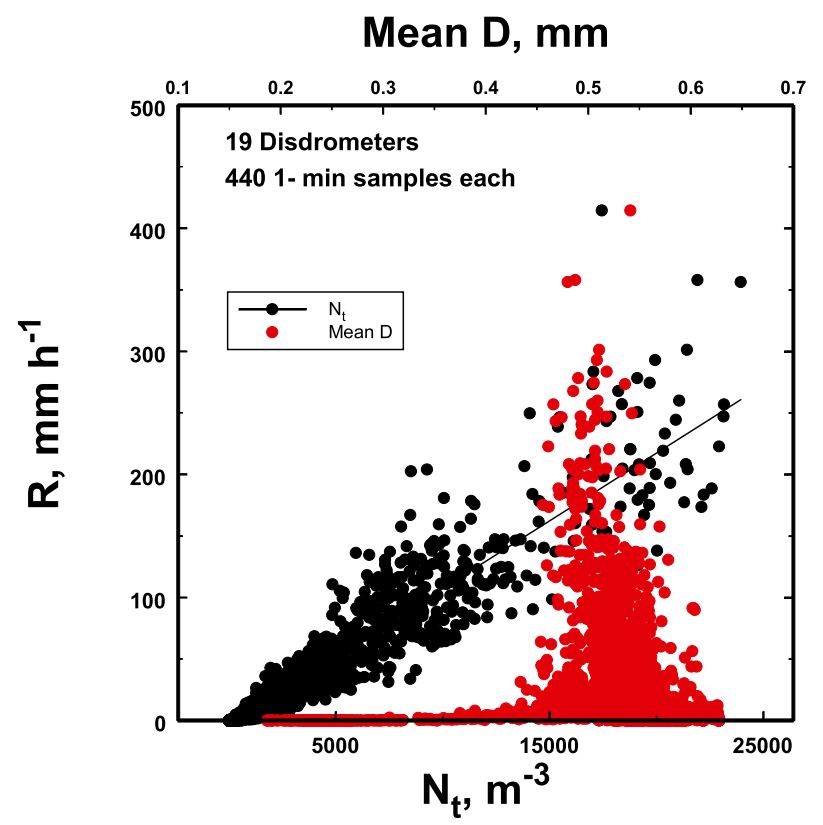

FIG. 17. Scatterplots of $R$ vs $N_{t}$ and vs $D$ at all the disdrometers for every minute showing the strong correlation between $N_{t}$ and $R$ as compared to the lack of correlation with $D$.

detectors' 440 temporal observations as well (Fig. 17). This is in strong contrast with conventional wisdom, which links changes in the rainfall rate to changes in the $P(D)$ (see Marshall and Palmer 1948, Sekhon and Srivastava 1971, and many others), which, in turn, imply power law relations between $R$ and $N_{t}$ as illustrated in Fig. 18. This suggests that it is reasonable to question the validity of drop size distribution powerlaw relations that do not satisfy a linear relation between $R$ and $N_{t}$. It also suggests that heterogeneous rain can be considered as an ensemble of homogeneous rain occurrences (Jameson and Kostinski 2001b) each with its own linear $R-N_{t}$ relation because in such a combination, linearity is preserved although the scatter increases. Thus, once again we see that powerlaw relations for statistically heterogeneous rain are only statistical fits, not physically meaningful relations among variables (Jameson and Kostinski 2001b).

Apparently, a linear relation between $R$ and $N_{t}$ arises in statistically heterogeneous rain because once the observations stop, there remains an average $D^{3} V_{t}$ that implies an associated average $P(D)$ such that $R \propto N_{t}$. In that sense, different $R \propto N_{t}$ relations imply different average $P(D)$. However, rather than being exact as in the statistically homogeneous case, there can be significant scatter around the net $R-N_{t}$ relation for heterogeneous rain because $P(D)$ changes during the observations and the results then depend upon when the measurements cease. 


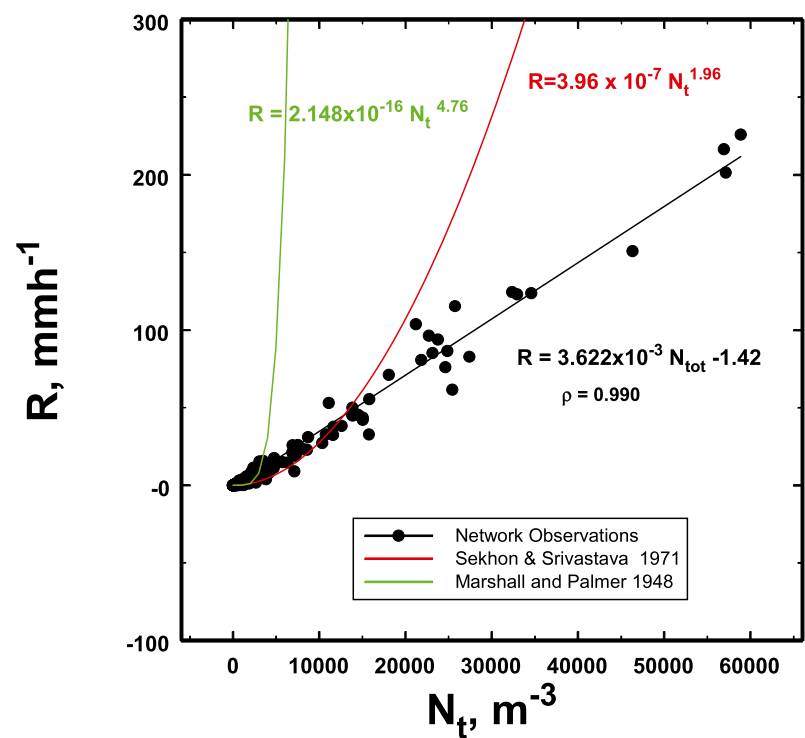

FIG. 18. Network-1-min-average $R$ vs network-average $N_{t}$ for the entire $1-440 \mathrm{~min}$. The red line is the relation derived based upon the radar study of Sekhon and Srivastava (1971) showing the effects of small-drop truncation by the radar. The green line is the powerlaw fit corresponding to the Marshall and Palmer (1948) distributions. The poor performance of power laws is discussed in the text.

Using the same argument as above, one might then expect a similar $R-N_{t}$ relation between the spatial averages over a network as indeed appears in Fig. 18. But will it be the same relation as that for a single instrument temporal average? In general, the answer is "no" as illustrated in Fig. 19. While the relation for network averages exists with a substantial correlation, the temporal relations for individual detectors can vary considerably both among themselves (i.e., depending upon location) and from the relation for spatial averages. That is, the average $P(D)$ values are not the same. Hence, in general, temporal- and spatial-average $P(D)$ cannot be considered to be equivalent nor will their variability be identical. While this result may seem trivial, this represents a direct confirmation of the often-neglected difference between temporal and spatial averaging and the danger of oversubscribing to the advection velocity transformation. What is happening even over this small area and even over $7 \mathrm{~h}$ is not necessarily well represented by measurements using a single instrument as Fig. 19 shows.

\section{Summary}

In this study, preliminary observations using a new network of 19 optical disdrometers are analyzed in both time and space for both convective and stratiform rain. Although this study cannot be considered

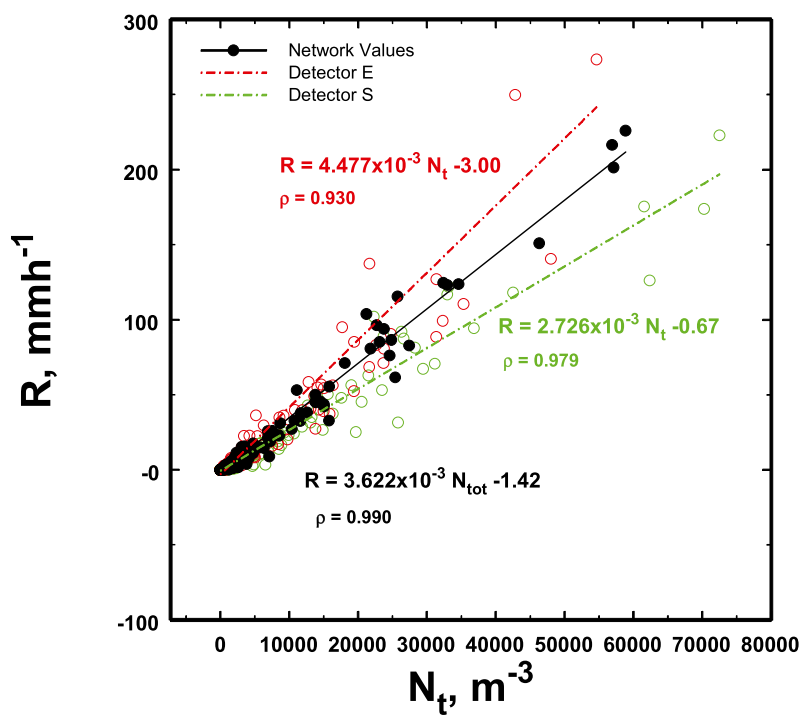

FIG. 19. A plot of the network-spatial-average $N_{t}$ and $R$ as well as plots of the time series for $N_{t}$ and $R$ for two individual optical disdrometers highlighting the differences between spatial and temporal relations that can occur.

complete in any sense, these data have already revealed a few interesting phenomena. One of the most important is the clarification of the different roles that space and time play in rainfall variability. That is, even over $900 \mathrm{~m}^{2}$, there is significant remnant spatial variability even after up to $440 \mathrm{~min}$ of rain particularly in more convective rain. In the one example of the most convective rain with peak rainfall rates approaching $250 \mathrm{~mm} \mathrm{~h}^{-1}$, the residual spatial variability is dominated by spatial clustering over all the drops, but its importance increases as the drop size increases for $D>1 \mathrm{~mm}$ until it exceeds $90 \%$ of the total variability for $D>2 \mathrm{~mm}$. This contrasts markedly with the more stratiform rain example in which the temporal clustering dominates exclusively over all sizes except for the very largest drops observed having $D>2.5 \mathrm{~mm}$, most likely associated with weak convective elements passing through the background of the more stratiform rain.

In another example, there is a mixture of the two types of rainfall-that is, a background of smaller drops, spatially more uniform but embedding spatially clustered drops of sizes larger than 1-mm size occasionally passing through the background rain.

What this points out is that there can be both temporal and spatial clustering each differently affecting drops of different sizes. Thus, there is no equivalency between spatial and temporal clustering. Both are important at different times and at different locations depending on the drop size and the meteorology. 
In addition, an attempt was made to find a simple advection velocity transformation between $\mathrm{CI}$ and SPCF. CIs are well fit by decreasing exponential functions. On the other hand, SPCFs can be fit using a wide variety of nonexponential as well as exponential functions. However, these latter exponentials are not simple decreasing exponentials like those for CI, but, rather, are decreasing stretched exponential functions of varying powers of separation distances. This suggests that there is no simple linear advection velocity transformation between CIs and SPCFs, at least for these data. However, the fits to SPCFs are all uncertain $(\rho=$ 0.10 ) so that more data must be analyzed for a more convincing conclusion in the future.

While there remains a wide variety of other problems to be addressed in future research, one example points to the advantage of having a number of instruments sampling over the modest area of $10000 \mathrm{~m}^{2}$. For spatial and temporal variability even over such small domains, the data can be combined to explore network-wide characteristics of the rain. Specifically, just as for time series measurements by a single disdrometers, a network of disdrometers also reveals a linear relation between $R$ and $N_{t}$ for the 1-min network-average values. This is in strong contrast with conventional wisdom, which links changes in the rainfall rate to changes in $P(D)$, which, in turn, imply power-law relations between $R$ and $N_{t}$ rather than the linear relations that we observe. Moreover, the network relation differs from those for time series observations by individual instruments. Because $R$ and $N_{t}$ are connected by $P(D)$ expected or average weighted moment $\left(D^{3} V_{t}\right)$, where $V_{t}$ is the terminal fall speed of drop of diameter $D$, this then implies that, in general, temporal- and spatial-average $P(D)$ values are not equivalent. While this result may seem trivial, this finding and the other results presented above represent a direct confirmation of the oftenneglected differences between temporal and spatial averages and the overuse of the advection velocity transformation.

Acknowledgments. This work was supported by the National Science Foundation (NSF) under Grant AGS130087, as well as by the United States Social Security Administration. Support for ML came from the National Science Foundation under Grant AGS1230240. Support for AK came from NSF Grant AGS111916. The authors are also especially grateful to the students of Prof. Larsen-namely Joerael Harris, Robert Lemasters, Katelyn O’Dell, Joshua Teves, and Michael Chute-who diligently worked to make this network a functioning reality.

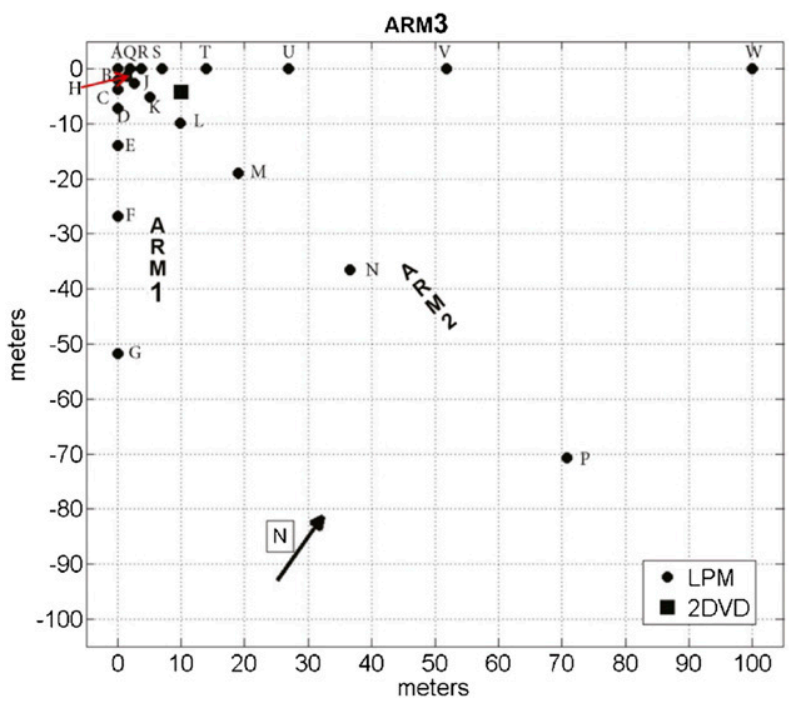

FIG. A1. A schematic of the layout of the LPM (optical disdrometers) network with the letters referencing specific instruments as further discussed in the text. The 2DVD is indicated by the box. The origin of the network is taken to be at detector A in the upper-left corner. Arms refer to specific linear arrays of instruments as referenced in the text at times.

\section{APPENDIX}

\section{A Description of the College of Charleston Disdrometer Network}

The network consists of 21 Thies laser precipitation monitors (LPMs), in conjunction with a Joanneum compact two-dimensional video disdrometer (2DVD). The array is located at historic "Dixie Plantation" near Hollywood, South Carolina; this property (owned by the College of Charleston Foundation) is used for a variety of ecological, educational, and research purposes. The site is located at $32^{\circ} 44^{\prime} 26^{\prime \prime} \mathrm{N}, 80^{\circ} 10^{\prime} 36^{\prime \prime} \mathrm{W}$. The general structure of the array can be seen schematically in Fig. A1, satellite imagery of the currently completed array is shown in Fig. A2, and a photograph of part of the array is shown in Fig. A3.

The instrument layout shown in Fig. A1 was designed to develop a dense network with distinct spatial separations. This layout contrasts with the usual grid setup, which collects a lot of information at only one particular separation distance but then abandons information on many other scales. By using logarithmic spacing, however, spatial scales from approximately 1 to $100 \mathrm{~m}$ can be explored simultaneously. With the addition of the compact 2DVD (which is capable of resolving spatial information smaller than $1 \mathrm{~mm}$ ), this array then allows us to investigate rainfall spatial variability through five orders of magnitude, most of which have not been 

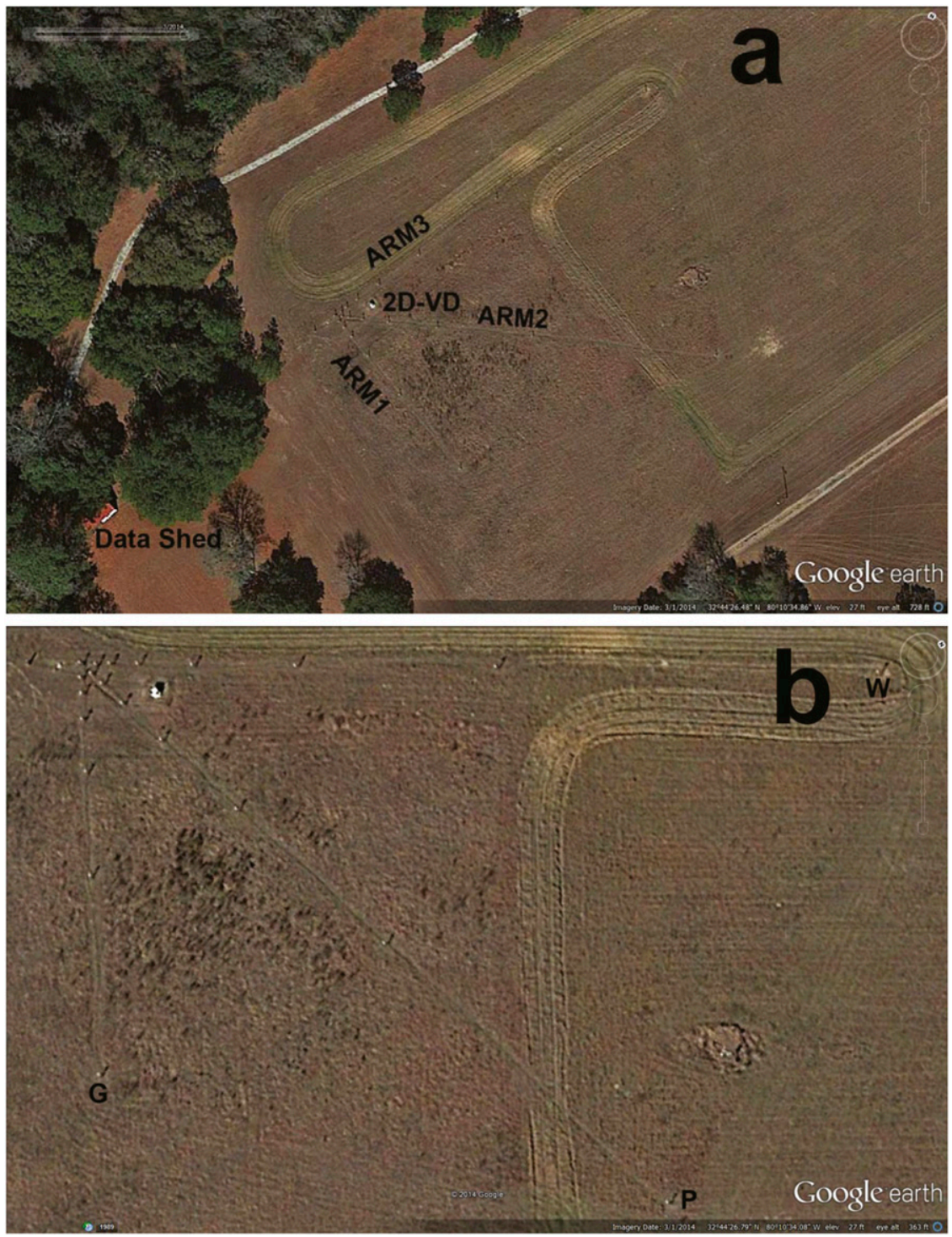

FIG. A2. (a) A Google Earth overview of the network and (b) a closer view of the layout of the disdrometers. Shadows extend from the optical disdrometers with letters denoting the detectors farthest from the origin along each arm.

extensively explored in past studies. Because of the logarithmic spacing the 21 LPMs allow for 66 distinct interdetector distances, allowing for reasonably dense resolution of spatial correlation functions as shown in the main text.

Figure A2 shows two views of the array site, taken from satellite imagery gathered on 1 March 2014. Figure A2a provides an overview not only of the full array but also of the surrounding tree cover and the location of the building with the data acquisition computers. The origin of the array was chosen far enough away from the tree cover to ensure no rain that should be detected hits the surrounding trees. The data is transmitted through cables back to the building with the red roof in the lower-left-hand corner of the image.

The more-zoomed image of the site (Fig. A2b) shows the individual detectors arm 3 runs from left to right across the top of the image. One can also see an additional post several meters to the left of the array origin and a data junction box about halfway between the extra post and the array origin. The 2DVD (installed in December) is the white spot between arms 2 and 3 near the origin of the array. The roughly circular patch between the ends of arms 2 and 3 contains a Davis VantagePro 2 weather station with a tipping-bucket rain gauge (not used in this study). 


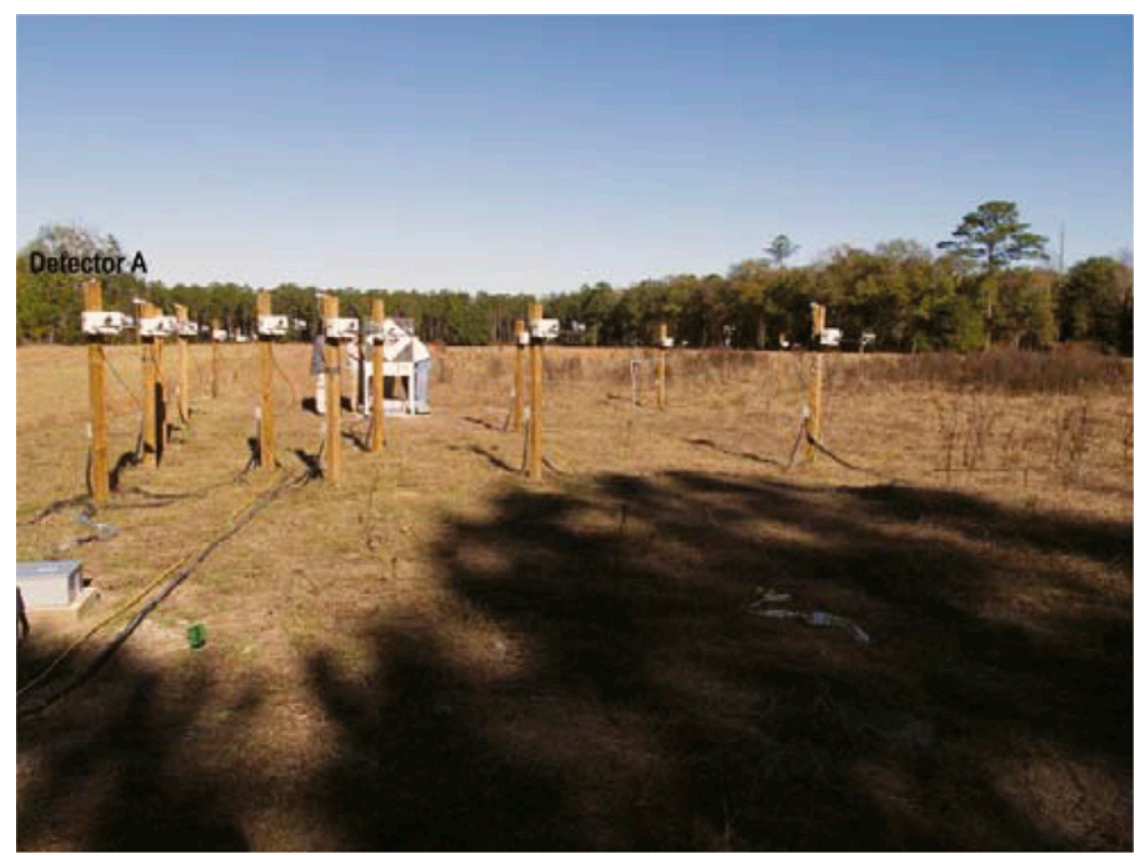

FIG. A3. A photograph of the layout of the LPM (optical disdrometers) network. The origin of the network is taken to be at detector A indicated at the left. Arm 3 is the leftmost line of detectors. The white box is the 2DVD toward the left of center.

Figure $\mathrm{A} 3$ is a photograph of the area near the network origin. Each LPM is mounted approximately $5 \mathrm{ft}(1.5 \mathrm{~m})$ above the ground on a $10-\mathrm{ft}(3.0 \mathrm{~m})$ pole [sunk $4 \mathrm{ft}(1.2 \mathrm{~m})$ into the ground]. The power for each detector comes from power supplies in the data acquisition building.

Construction of the network was begun in May 2013. Each LPM was calibrated in a separate indoor laboratory and moved to the field site. Instruments came online during the interval between September and December 2013, with the 2DVD being the last-installed piece in mid-December 2013. This study uses data taken by 19 operational LPMs during the instrument deployment stage. (LPMs L and M had glitches in the wiring that were not rectified until early December.)

The Thies LPM instruments were characterized in detail by Frasson et al. (2011) and have been used in a number of other studies including Brawn and Upton (2008) and Fernández-Raga et al. (2009, 2010). Optical disdrometers are well recognized as useful tools for characterizing drop size distributions (e.g., LöfflerMang and Yuter 2002; Tokay et al. 2001). The instruments are infrared occlusion instruments that can be run in several separate modes. For this study, the instruments were run in their default mode, associated with 1-min integrations; in this mode, the device reports a spectrum each minute indicating how many droplets were detected in each of 22 disjoint drop size bins and 20 disjoint velocity bins (thus, each drop is characterized as belonging to 1 of 440 different categories). These instruments do not appear in the literature as often as some other optical or impact disdrometers, but they were utilized in the construction of this array because they were among the most affordable devices for resolving 1-min raindrop size spectra. The known issues associated with particle sizing were mitigated to the greatest degree possible by verifying consistent performance in the laboratory before deployment and using identical instruments throughout the array. Moreover, measurements by all of the instruments were compared to minimize the inclusion of questionable behavior.

The devices are naturally synchronized; all devices were turned on simultaneously since they are run with the same power supply. Although these devices are intended to be very low maintenance, the acquisition of data is reset every week to ensure minimal temporal drift; empirical estimation from this process suggests a relative shift of less than $1{\mathrm{~s} w e e \mathrm{k}^{-1}}$ among all detectors. During the weekly maintenance activity, the devices are confirmed to be level, free of debris/insects, and recording properly.

\section{REFERENCES}

Anderson, A., and A. Kostinski, 2010: Reversible record braking and variability: Temperature distributions across the globe. J. Appl. Meteor. Climatol., 49, 1681-1691, doi:10.1175/2010JAMC2407.1. 
Baker, B., and R. P. Lawson, 2010: Analysis of tools used to quantify droplet clustering in clouds. J. Atmos. Sci., 67, 33553367, doi:10.1175/2010JAS3409.1.

Brawn, D., and G. Upton, 2008: On the measurement of atmospheric gamma drop-size distributions. Atmos. Sci. Lett., 9, 245-247, doi:10.1002/asl.198.

Caracciolo, C., M. Napoli, F. Porcu, F. Prodi, S. Dietrich, C. Zanchi, and S. Orlandini, 2012: Raindrop size distribution and soil erosion. J. Irrig. Drain. Eng., 138, 461-469, doi:10.1061/(ASCE)IR.1943-4774.0000412.

Chaumat, L., and J. L. Brenguier, 2001: Droplet spectra broadening in cumulus clouds. Part II: Microscale droplet concentration heterogeneities. J. Atmos. Sci., 58, 642-654, doi:10.1175/ 1520-0469(2001)058<0642:DSBICC >2.0.CO;2.

Fernández-Raga, M., A. Castro, C. Palencia, A. Calvo, and R. Fraile, 2009: Rain events on 22 October 2006 in León (Spain): Drop size spectra. Atmos. Res., 93, 619-635, doi:10.1016/ j.atmosres.2008.09.035.

_- and Coauthors, 2010: The kinetic energy of rain measured with an optical disdrometer: An application to splash erosion. Atmos. Res., 96, 225-240, doi:10.1016/j.atmosres.2009.07.013.

Frasson, R. P. M., L. K. da Cunha, and W. F. Krajewski, 2011: Assessment of the Thies optical disdrometer performance. Atmos. Res., 101, 237-255, doi:10.1016/j.atmosres.2011.02.014.

Green, H. S., 1969: The Molecular Theory of Fluids. Dover, 737 pp.

Haralick, R. M., and L. G. Shapiro, 1992: Computer and Robot Vision. Vol. II. Addison-Wesley, 630 pp.

Jaffrain, J., and A. Berne, 2012: Quantification of the small-scale spatial structure of the raindrop size distribution from a network of disdrometers. J. Appl. Meteor. Climatol., 51, 941-953, doi:10.1175/JAMC-D-11-0136.1.

- A. Studzinski, and A. Berne, 2011: A network of disdrometers to quantify the small-scale variability of the raindrop size distribution. Water Resour. Res., 47, W00H06, doi:10.1029/2010WR009872.

Jameson, A. R., 2008: Radar observations of rainfall variability using non-Rayleigh signal fluctuations. J. Appl. Meteor. Climatol., 47, 607-619, doi:10.1175/2007JAMC1630.1.

_ 2014: A Bayesian method for upsizing disdrometer drop size counts for rain physics studies and areal applications. IEEE Trans. Geosci. Remote Sens., 53, 335-343, doi:10.1109/ TGRS.2014.2322092.

— caused by relative motion during measurement. J. Appl. Meteor., 35, 1846-1859, doi:10.1175/1520-0450(1996)035<1846: NRSSCB $>2.0 . \mathrm{CO} ; 2$.

$\longrightarrow$, and - 1999: Fluctuation properties of precipitation. Part V: Distribution of rain rates-Theory and observations in clustered rain. J. Atmos. Sci., 56, 3920-3932, doi:10.1175/ 1520-0469(1999)056<3920:FPOPPV > 2.0.CO;2.

— , and - 2000: Fluctuation properties of precipitation. Part VI: Observations of hyper-fine clustering and drop size distribution structures in three-dimensional rain. J. Atmos. Sci., 57, 373-388, doi:10.1175/1520-0469(2000)057<0373: FPOPPV $>2.0 . \mathrm{CO} ; 2$.

— , and — 2001a: What is a raindrop size distribution? Bull. Amer. Meteor. Soc., 82, 1169-1177, doi:10.1175/ 1520-0477(2001)082<1169:WIARSD > 2.3.CO;2.

- and $\longrightarrow, 2001 \mathrm{~b}$ : Reconsideration of the physical and empirical origins of $Z-R$ relations in radar meteorology. Quart. J. Roy. Meteor. Soc., 127, 517-538, doi:10.1002/qj.49712757214.

— , and - 2002: When is rain steady? J. Appl. Meteor., 41, 8390, doi:10.1175/1520-0450(2002)041<0083:WIRS>2.0.CO;2.
- , and - 2008: The effect of clustering on the uncertainty of differential reflectivity measurements. J. Appl. Meteor. Climatol., 47, 2816-2827, doi:10.1175/2008JAMC1860.1.

$\longrightarrow$, and —, 2010: Partially coherent backscatter in radar observations of precipitation. J. Atmos. Sci., 67, 1928-1946, doi:10.1175/2010JAS3336.1.

Kinnell, P. I. A., 2005: Raindrop-impact-induced erosion processes and prediction: A review. Hydrol. Processes, 19, 2815-2844, doi:10.1002/hyp.5788.

Kostinski, A. B., and A. R. Jameson, 1997: Fluctuation properties of precipitation. Part I: On deviations of single-size counts from the Poisson distribution. J. Atmos. Sci., 54, 2174-2186, doi:10.1175/1520-0469(1997)054<2174:FPOPPI >2.0.CO;2.

, and - 2000: On the spatial distribution of cloud particles. J. Atmos. Sci., 57, 901-915, doi:10.1175/1520-0469(2000)057<0901: OTSDOC $>2.0 . \mathrm{CO} ; 2$

- M. L. Larsen, and A. R. Jameson, 2006: The texture of rain: Exploring stochastic micro-structure at small scales. J. Hydrol., 328, 38-45, doi:10.1016/j.jhydrol.2005.11.035.

Koutsoyiannis, D., 2006: An entropic-stochastic representation of rainfall intermittency: The origin of clustering and persistence. Water Resour. Res., 42, W01401, doi:10.1029/2005WR004175.

Krajewski, W. F., G. J. Ciach, and E. Habib, 2003: An analysis of small-scale rainfall variability in different climatic regimes. Hydrol. Sci. J., 48, 151-162, doi:10.1623/hysj.48.2.151.44694.

Kundu, P. K., and J. E. Travis, 2013: A stochastic fractional dynamics model of space-time variability of rain. J. Geophys. Res. Atmos., 118, 102277-10 295, doi:10.1002/jgrd.50723.

Landau, L. D., and E. M. Lifshitz, 1980: Statistical Physics. Pergamon Press, $687 \mathrm{pp}$.

Larsen, M. L., 2012: Scale localization of cloud particle clustering statistics. J. Atmos. Sci., 69, 3277-3289, doi:10.1175/ JAS-D-12-02.1.

—, A. Clark, M. Noffke, G. Saltzgaber, and A. Steele, 2010: Identifying the scaling properties of rainfall accumulation as measured by a rain gage network. Atmos. Res., 96, 149-158, doi:10.1016/j.atmosres.2009.12.008.

, A. B. Kostinski, and A. R. Jameson, 2014: Evidence supporting the existence of superterminal raindrops. Geophys. Res. Lett., 41, 6914-6918, doi:10.1002/2014GL061397.

Li, B., A. Murthi, K. P. Bowman, G. R. North, M. G. Genton, and M. Sherman, 2009: Statistical tests of Taylor's hypothesis: An application to precipitation fields. J. Hydrometeor., 10, 254265, doi:10.1175/2008JHM1009.1.

Löffler-Mang, M., and S. Yuter, 2002: Particle type, velocity, and size distribution measurements with the PARSIVEL optical disdrometer. Proc. GPM Workshop, Seattle, WA, NASA, 23-25.

Marshall, J. S., and W. M. Palmer, 1948: The distributions of raindrops with size. J. Meteor., 5, 165-166, doi:10.1175/ 1520-0469(1948)005<0165:TDORWS $>2.0$. CO;2.

Molini, A., G. Katul, and A. Porporato, 2009: Revisiting rainfall clustering and intermittency across different climatic regimes. Water Resour. Res., 45, W11403, doi:10.1029/ 2008WR007352.

Montero-Martínez, G., A. B. Kostinski, R. A. Shaw, and F. GarcíaGarcía, 2009: Do all raindrops fall at terminal speed? Geophys. Res. Lett., 36, L11818, doi:10.1029/2008GL037111.

Mood, A. M., F. A. Graybill, and D. C. Boes, 1974: Introduction to the Theory of Statistics. 3rd ed. McGraw-Hill, 564 pp.

Ornstein, L., and F. Zernike, 1914: Accidental deviations of density and opalescence at the critical point of a single substance. Proc. Roy. Neth. Acad. Arts Sci., 17, 793-806. 
Peebles, P. J. E., 1993: Principles of Physical Cosmology. Princeton University Press, 709 pp.

Pimentel, D., and Coauthors, 1995: Environmental and economic costs of soil erosion and conservation benefits. Science, 267, 1117-1123, doi:10.1126/science.267.5201.1117.

Sekhon, R. S., and R. C. Srivastava, 1971: Doppler radar observations of drop-size distributions in a thunderstorm. J. Atmos. Sci., 28, 983994, doi:10.1175/1520-0469(1971)028<0983:DROODS >2.0.CO;2.

Smith, J. A., E. Hui, M. Steiner, M. L. Baeck, W. F. Krajewski, and A. A. Ntelekos, 2009: Variability of rainfall rate and raindrop size distributions in heavy rain. Water Resour. Res., 45, W04430, doi:10.1029/2008WR006840.

Steiner, M., J. A. Smith, and R. Uijlenhoet, 2004: A microphysical interpretation of radar reflectivity-rain rate relationships. J. Atmos. Sci., 61, 1114-1131, doi:10.1175/1520-0469(2004)061<1114: AMIORR $>2.0 . \mathrm{CO} ; 2$.
Tokay, A., and P. G. Bashor, 2010: An experimental study of small-scale variability of raindrop size distribution. J. Appl. Meteor. Climatol., 49, 2348-2365, doi:10.1175/ 2010JAMC2269.1.

— A. Kruger, and W. F. Krajewski, 2001: Comparison of drop size distribution measurements by impact and optical disdrometers. J. Appl. Meteor., 40, 2083-2097, doi:10.1175/ 1520-0450(2001)040<2083:CODSDM > 2.0.CO;2.

_ P. G. Bashor, and K. R. Wolff, 2005: Error characteristics of rainfall measurements by co-located Joss-Waldvogel disdrometers. J. Tech., 22, 513-527.

Uri, N. D., and J. A. Lewis, 1999: Agriculture and the dynamics of soil erosion in the United States. J. Sustainable Agric., 14, 6382, doi:10.1300/J064v14n02_07.

Watson, D. F., 1994: NNGRIDR: An Implementation of Natural Neighbor Interpolation. David Watson, 170 pp. 